BULLETIN (New Series) OF THE

AMERICAN MATHEMATICAL SOCIETY

Volume 48, Number 3, July 2011, Pages 409-455

S 0273-0979(2011)01338-6

Article electronically published on April 25, 2011

\title{
SYMPLECTIC THEORY OF COMPLETELY INTEGRABLE HAMILTONIAN SYSTEMS
}

\author{
ÁLVARO PELAYO AND SAN VŨ NGỌC \\ In memory of Professor Johannes (Hans) J. Duistermaat (1942-2010)
}

\begin{abstract}
This paper explains the recent developments on the symplectic theory of Hamiltonian completely integrable systems on symplectic 4-manifolds, compact or not. One fundamental ingredient of these developments has been the understanding of singular affine structures. These developments make use of results obtained by many authors in the second half of the twentieth century, notably Arnold, Duistermaat, and Eliasson; we also give a concise survey of this work. As a motivation, we present a collection of remarkable results proved in the early and mid-1980s in the theory of Hamiltonian Lie group actions by Atiyah, Guillemin and Sternberg, and Delzant among others, and which inspired many people, including the authors, to work on more general Hamiltonian systems. The paper concludes with a discussion of a spectral conjecture for quantum integrable systems.
\end{abstract}

\section{INTRODUCTION}

In the mathematical theory of conservative systems of differential equations, one finds cases that are solvable in some sense, or integrable, which enables one to study their dynamical behavior using differential geometric and Lie 1 theoretic methods, in particular the theory of Lie group actions on symplectic manifolds. Integrable systems are a fundamental class of "explicitly solvable" dynamical systems of current interest in differential and algebraic geometry, representation theory, analysis, and physics. Their study usually combines ideas from several areas of mathematics, notably partial differential equations, microlocal analysis, Lie theory, symplectic geometry, and representation theory. In this paper we focus on finite-dimensional completely integrable Hamiltonian systems (sometimes called "Liouville integrable systems") in the context of symplectic geometry.

Many authors have studied dynamical problems for centuries. Galileo made great advances in the subject in the late sixteenth and early seventeenth centuries, and formulated the "laws of falling bodies". An important contribution was made by Huygens in the seventeenth century, who studied in detail the spherical pendulum,

Received by the editors July 16, 2010, and, in revised form, November 29, 2010, and March $21,2011$.

2010 Mathematics Subject Classification. Primary 37J35; Secondary 37J05, 37J15, 53D35, 37K10, 53D20, 14H70.

${ }^{1}$ Sophus Lie has been one of the most influential figures in differential geometry. Many modern notions of differential geometry were known to Lie in some form, including the notion of symplectic manifolds, symplectic and Hamiltonian vector fields, transformation (=Lie) groups, and (in a particular instance) momentum maps.

(C)2011 American Mathematical Society 
a simple but fundamental example. Galileo's ideas were generalized and reformulated by William Hamilton (1805-1865) using symplectic geometry, who said: "the theoretical development of the laws of motion of bodies is a problem of such interest and importance that it has engaged the attention of all the eminent mathematicians since the invention of the dynamics as a mathematical science by Galileo, and especially since the wonderful extension which was given to that science by Newton" (1834, cf. J. R. Taylor [101, p. 237]). Many of the modern notions in the mathematical theory of dynamical systems date back to the late nineteenth century and the twentieth century, to the works of Poincaré, Lyapunov, Birkhoff, Siegel, and the Russian school in the qualitative theory of ordinary differential equations.

A completely integrable Hamiltonian system may be given by the following data 2 (1) a $2 n$-dimensional smooth manifold $M$ equipped with a symplectic form, and (2) $n$ smooth functions $f_{1}, \ldots, f_{n}: M \rightarrow \mathbb{R}$ which generate vector fields that are pairwise linearly independent at almost every point, and which Poisson-commute. In local symplectic coordinates, this commuting condition amounts to the vanishing of partial differential equations involving the $f_{i}$; see, e.g., Section 6 . Many times we will omit the word "Hamiltonian" and refer simply to "completely integrable systems". A completely integrable system has a singularity at every point where this linear independence fails to hold. It is precisely at the singularities where the most interesting, and most complicated, dynamical features of the system are displayed. An important class of completely integrable systems, with well-behaved singularities, are those given by Hamiltonian $n$-torus actions on symplectic $2 n$ manifolds. These actions have a momentum map with $n$ components $f_{1}, \ldots, f_{n}$, and these components always form a completely integrable system. A remarkable structure theory by Atiyah [6], Guillemin and Sternberg [56], and Delzant [30] exists for these systems, which are usually referred to as toric systems.

The study of completely integrable Hamiltonian systems is a vast and active research area. Two motivations to study such systems come from (i) KolmogorovArnold-Moser (KAM) theory: since integrable systems are "solvable" in a precise sense, one expects to find valuable information about the behavior of dynamical systems that are obtained by small perturbations of them, and then the powerful KAM theory comes into play (see de la Llave's article 29 for a summary of the main ideas of KAM theory) to deal with the properties of the perturbations (persistence of quasi-periodic motions); (ii) the theory of singularities of fibrations $\left(f_{1}, \ldots, f_{n}\right): M \rightarrow \mathbb{R}$ by the Fomenko school [12]: the Fomenko school has developed powerful and far-reaching methods to study the topology of singularities of integrable systems. It is interesting to notice that there is a relation between these two motivations, which has been explored recently by Dullin and Vũ Ngọc [39, 40, and Nguyên Tiên Zung [114, 117.

In the present article we give an overview of our perspective of the current state of the art of the symplectic geometry of completely integrable systems, with a particular emphasis on the the recent developments on semitoric integrable systems in dimension four. Before this, we briefly review several preceding fundamental results due to Arnold, Atiyah, Carathéodory, Darboux, Delzant, Duistermaat, Dufour, Eliasson, Guillemin, Liouville, Mineur, Molino, Sternberg, Toulet, and Nguyên Tiên Zung, some of which are key ingredients in the symplectic theory of semitoric integrable systems. This article does not intend to be comprehensive in any way,

${ }^{2}$ We will explain this definition in detail later, starting with the most basic notions. 
but rather it is meant to be a fast overview of the current research in the subject. We hope we will convey some of the developments which we consider most representative. Our point of view is that of local phase-space analysis - it advocates for the use of local normal forms and sheaf theoretic methods to prove global results by gluing local pieces.

Some of the current activity on integrable systems is concerned with a question of high interest to applied and pure mathematicians and physicists. The question is whether one can reconstruct an integrable system that one does not know a priori from observing some of its properties; consider, e.g., Kac's famous question: Can you hear the shape of a drum? Kac's question in the context of integrable systems can be formulated in the following way: Can a completely integrable system be recovered from the joint spectrum of the corresponding quantized integrable system? Such a question is a strong motivation for the symplectic study of integrable systems. Indeed, a conjugation by a unitary operator is the natural quantization of a symplectic diffeomorphism. Therefore, a good inverse spectral theory must rely upon a tractable symplectic classification. Symplectic geometry is the main goal of this article. Occasionally, and particularly in the last section, we will make some further comments on quantization; for a basic reference on the so called geometric quantization, see for example Kostant and Pelayo 64.

The authors have recently given a global symplectic classification of integrable systems with two degrees of freedom 3 no hyperbolic singularities and for which one component of the system is a $2 \pi$-periodic Hamiltonian 87, 88; these systems are called semitoric. We devote Sections 6, 7 and 8 this paper to explain this symplectic classification. This symplectic classification of semitoric integrable systems described in this paper prepares the ground for answering Kac's question in the context of quantum semitoric completely integrable systems.

Semitoric systems form an important class of integrable systems, commonly found in simple physical models; a semitoric system can be viewed as a Hamiltonian system in the presence of circular symmetry. Perhaps the simplest example of a noncompact nontoric semitoric system is the coupled spin-oscillator model $S^{2} \times \mathbb{R}^{2}$ described in [108, Section 6.2], where $S^{2}$ is viewed as the unit sphere in $\mathbb{R}^{3}$ with coordinates $(x, y, z)$, and the second factor $\mathbb{R}^{2}$ is equipped with coordinates $(u, v)$, equipped with the Hamiltonians $J:=\left(u^{2}+v^{2}\right) / 2+z$ and $H:=\frac{1}{2}(u x+v y)$. Here $S^{2}$ and $\mathbb{R}^{2}$ are equipped with the standard area forms, and $S^{2} \times \mathbb{R}^{2}$ with the product symplectic form. The authors have carried out the quantization of this model in 89. Another interesting semiclassical study of this system can be found in 9 .

In the aforementioned papers we combine techniques from classical differential geometry, semiclassical analysis, and Lie theory; these works are representative of our core belief that one can make definite progress in understanding the symplectic and spectral theory of integrable systems by combining techniques and ideas from these areas. This symplectic work in turn generalizes the celebrated theory of Hamiltonian Lie group actions by Atiyah, Benoist, Delzant, Guillemin, Kirwan, Sternberg, and others, to completely integrable systems. It is also intimately connected with several previous works [32, 33, 34, 35, 61, 83, 107, 108.

While major progress has been made in recent times by many authors, the theory of integrable systems in symplectic geometry is far from complete at the present

\footnotetext{
${ }^{3}$ The number of degrees of freedom is half the dimension of the symplectic manifold.
} 
time, even in the case of integrable systems with two degrees of freedom. For example, it remains for us to understand the symplectic theory of integrable systems on 4-manifolds when one allows hyperbolic singularities to occur. The presence of hyperbolic singularities has a global effect on the system which makes describing a set of global invariants difficult 4 We do not know at this time if this is even a feasible problem or whether one can expect to give a reasonable classification extending the case where no hyperbolic singularities occur. The classification of semitoric systems of the authors is expressed in terms of several invariants, one of which is a class of polygons (the "polygon invariant"). It seems conceivable that in the presence of hyperbolic singularities, this polygon invariant should be replaced by a foliation with affine leaves with branches. Foliation type invariants are of high interest in the fileds of dynamical systems and symplectic geometry. Finite dimensional integrable Hamiltonian systems, in particular semitoric systems [87, 88, play a prominent role at the intersection of these two fields.

Foliation and foliation invariants have been introduced and studied from many different viewpoints and in many different contexts within symplectic geometry. Some of these works are, for example, Cushman and Bates's book 27] from the angle of classical mechanics; Bolsinov and Fomenko's book 12 from a singularity theory angle; Pelayo [83, Sections 2 and 3] in the context of symplectic manifolds with symplectic orbits and flat connections; Vũ Ngọc [107] in the context of focus-focus singularities (treated later in this paper); Nguyên Tiên Zung [115, Section 7] in the context of topology of singular Lagrangian foliations; and Branham and Hofer's recent interesting survey [14, Section 4] from the point of view of holomorphic curves. A beautiful aspect of the study of integrable systems is how one sees a fruitful interaction of methods and ideas from different, sometimes seemingly unrelated, areas.

Moreover, the current theory allows us to understand semitoric systems with controlled behavior at infinity; precisely, this means that the $2 \pi$-periodic Hamiltonian is a proper map (a paper in the works 85 is expected to address this case). The general case is open, however.

Shedding light onto these two questions would bring us a step closer to understanding the symplectic geometry of general completely integrable systems with two degrees of freedom in dimension 4, which we view as one of the major and longstanding unsolved problems in geometry and dynamics (and to which many people have made contributions, several of which are mentioned in the present paper). In addition, answers to these questions constitute another required step towards a quantum theory of integrable systems on symplectic manifolds.

One can find integrable systems in different areas of mathematics and physics. For example, in the context of algebraic geometry, a semitoric system naturally gives a toric fibration with singularities, and its base space becomes endowed with a singular integral affine structure. Remarkably, these singular affine structures are of key importance in various parts of symplectic topology, mirror symmetry, and algebraic geometry - for example they play a central role in the work of Kontsevich and Soibelman [67]; cf. Section 9.2 for further details. Interesting semitoric systems also appear as relevant examples in the theory of symplectic quasi-states; see

\footnotetext{
${ }^{4}$ This starts with what we will call the "polygon invariant" and which encodes in some precise sense the affine structure induced by the system.
} 
Eliashberg and Polterovich [41, page 3]. Many aspects of the global theory of semitoric integrable systems may be understood in terms of singular affine structures, but we do not know at this time whether all of the invariants may be expressed in terms of singular affine structures (if this were the case, it would likely involve some asymptotic behavior).

For mathematicians semitoric systems are the next natural class of integrable systems to consider after toric systems. Semitoric systems exhibit a richer, less rigid behavior than toric systems. The mathematical theory of semitoric systems explained in the last few sections of this paper was preceded by a number of interesting works by physicists and chemists working on describing energy-momentum spectra of systems in the context of quantum molecular spectroscopy [44, 92, 19, 5]. Physicists and chemists were the first to become interested in semitoric systems. Semitoric systems appear naturally in the context of quantum chemistry. Many groups have been working on this topic, to name a few: Mark Child's group in Oxford (UK), Jonathan Tennyson's at University College London (UK), Frank De Lucia's at Ohio State University (USA), Boris Zhilinskii's at Dunkerque (France), and Marc Joyeux's at Grenoble (France).

These physicists and chemists have asked whether one can one give a finite collection of invariants characterizing systems of this nature. The theory of semitoric systems described in the present paper was largely motivated by this question, and fits into the broader realization in the physics and chemistry communities that symplectic invariants play a leading role in understanding a number of global questions in molecular spectroscopy - hence any mathematical discovery in this direction will be of interest outside of a pure mathematical context; see Stewart 98.

Direct applications of integrable systems can also be found in the theory of geometric phases, nonholonomic mechanics, rigid-body systems, fluid mechanics, elasticity theory, and plasma physics, and have been extensively carried out by many authors, including Marsden, Ratiu and their collaborators. The semiclassical aspects of integrable systems have been studied recently in the book [110] and the article [109. In the book 27] singular Lagrangian fibrations are treated from the point of view of classical mechanics. Finally, we would like to point out Bolsinov and Oshemkov's interesting review article [11, where for instance one can find information about hyperbolic singularities.

The structure of the paper is as follows. In Sections 2 3 , 4, and 5 we summarize some of the most important known results at a local and semiglobal level for Hamiltonian systems and motivate their study by presenting some influential results from the theory of Hamiltonian Lie group actions due to Atiyah, Guillemin and Sternberg, and Delzant. In Section 6 we introduce semitoric systems in dimension 4 and explain their convexity properties. In Sections 7 and 8 we introduce symplectic invariants for these systems and explain the recent global symplectic classification of semitoric systems given by the authors. In Section 9 we briefly discuss some open problems, with a particular emphasis on inverse spectral theory.

\section{SympleCtiC DYNAmics}

The unifying topic this paper is symplectic geometry, which is the mathematical language to clearly and concisely formulate problems in classical physics and to understand their quantum counterparts (see Marsden and Ratiu's classical textbook 76] for a treatment of classical mechanical systems). In the sense of Weinstein's 
creed, symplectic geometry is of interest as a series of remarkable "transforms" which connect it with several areas of semiclassical analysis, partial differential equations, and low-dimensional topology.

One may argue that symplectic manifolds are not the most general, or natural, setting for mechanics. In recent times some effort have been made to study Poisson structures, largely motivated by the study of coadjoint orbits. However only very few general results on integrable systems are known in the context of Poisson manifolds.

2.1. Symplectic manifolds. A symplectic form on a vector space $V$ is a nondegenerate, antisymmetric, bilinear map $V \times V \rightarrow \mathbb{R}$. A symplectic manifold is a pair $(M, \omega)$ where $M$ is a smooth manifold and $\omega$ is symplectic form on $M$, i.e., a smooth collection of symplectic forms $\omega_{p}$, one for each tangent space $\mathrm{T}_{p} M$, which is globally closed in the sense that the differential equation $\mathrm{d} \omega=0$ holds.

The simplest example of a symplectic manifold is probably a surface of genus $g$ with an area form. An important example is $\mathbb{R}^{2 n}$ with the form $\sum_{i=1}^{n} \mathrm{~d} x_{i} \wedge \mathrm{d} y_{i}$, where $\left(x_{1}, y_{1}, \ldots, x_{n}, y_{n}\right)$ are the coordinates in $\mathbb{R}^{2 n}$.

Symplectic manifolds are always even dimensional, so for example $S^{1}$ and $S^{3}$ cannot be symplectic. They are also orientable, where the volume form is given by $\omega \wedge \cdots(n$ times $) \cdots \wedge \omega=\omega^{n}$, if $\operatorname{dim} M=2 n$, so for example the Klein bottle is not a symplectic manifold. Moreover, symplectic manifolds are topologically "nontrivial" in the sense that if $M$ is compact, then the even-dimensional de Rham cohomology groups of $M$ are not trivial because $\left[\omega^{k}\right] \in \mathrm{H}_{\mathrm{dR}}^{2 k}(M)$ defines a nonvanishing cohomology class if $k \leq n$, i.e., the differential 2 -form $\omega^{k}$ is closed but not exact (the proof of this uses Stokes' theorem, and is not completely immediate). Therefore, the spheres $S^{4}, S^{6}, S^{8}, \ldots, S^{2 N}, \ldots$ cannot be symplectic. Symplectic manifolds were locally classified by Darboux and the end of the nineteenth century. He proved the following remarkable theorem.

Theorem 2.1 (Darboux [28]). Near each point in $(M, \omega)$ there exists coordinates $\left(x_{1}, y_{1}, \ldots, x_{n}, y_{n}\right)$ in which the symplectic form $\omega$ has the form $\omega=\sum_{i=1}^{n} \mathrm{~d} x_{i} \wedge$ $\mathrm{d} y_{i}$.

It follows from Darboux's theorem that symplectic manifolds have no local invariants other than the dimension. This is a fundamental difference with Riemannian geometry, where the curvature is a local invariant.

2.2. Dynamics of vector fields and torus actions. A smooth vector field $\mathcal{Y}$ on a symplectic manifold $(M, \omega)$ is symplectic if its flow preserves the symplectic form $\omega$; it is Hamiltonian if the system

$$
\omega(\mathcal{Y}, \cdot)=\mathrm{d} H \quad \text { (Hamilton's PDEs })
$$

has a smooth solution $H: M \rightarrow \mathbb{R}$. If so, we use the notation $\mathcal{Y}:=\mathcal{H}_{H}$ and call $H$ call the Hamiltonian or energy function.

For instance, the vector field $\frac{\partial}{\partial \theta}$ on $\mathbb{T}^{2}:=\left(S^{1}\right)^{2}$ is symplectic but not Hamiltonian ( $\theta$ is the coordinate on the first copy of $S^{1}$ in $\mathbb{T}^{2}$, for example). On the other hand, the vector field $\frac{\partial}{\partial \theta}$ on $S^{2}$ is Hamiltonian: $\frac{\partial}{\partial \theta}=\mathcal{H}_{H}$ with $H(\theta, h):=h$, where $(\theta, h)$ represents a point in the unit sphere $S^{2}$ of height $h$ measured from the plane $z=0$ and angle $\theta$ measured about the vertical axis; see Figure 2.1.

Suppose that we have local Darboux coordinates $\left(x_{1}, y_{1}, \ldots, x_{n}, y_{n}\right)$ near a point $m \in M$. Let $\gamma(t):=\left(x_{1}(t), y_{1}(t), \ldots, x_{n}(t), y_{n}(t)\right)$ be an integral curve of a smooth 
vector field $\mathcal{Y}$. Then $\mathcal{Y}=\mathcal{H}_{H}$ for a local smooth function $H$ if and only if

$$
\left\{\begin{array}{rrr}
\frac{\mathrm{d} y_{i}}{\mathrm{~d} t}(t) & =-\frac{\partial H}{\partial x_{i}}(\gamma(t)) \\
\frac{\mathrm{d} x_{i}}{\mathrm{~d} t}(t) & =\frac{\partial H}{\partial y_{i}}(\gamma(t))
\end{array}\right.
$$

It always holds that $H(\gamma(t))=$ const, i.e., that energy is conserved by motion (Noether's Principle). If $\mathcal{Y}$ is symplectic, these equations always have a local solution, but in order for the vector field $\mathcal{Y}$ to be Hamiltonian (globally), one must have that $\mathcal{Y}=\mathcal{H}_{H}$ on $M$, i.e., the function $H$ has to be the same on each local Darboux chart. From a more abstract point of view, this amounts to saying that the 1-form $\omega(\mathcal{Y}, \cdot)$ is always locally exact, but not necessarily globally exact. So the obstruction to being exact lies in the first de Rham cohomology group $\mathrm{H}_{\mathrm{dR}}^{1}(M)$ of $M$; if this group is trivial, then any smooth symplectic vector field on $M$ is Hamiltonian.

Now suppose that we have a torus $T \simeq \mathbb{T}^{k}:=\left(S^{1}\right)^{k}$, i.e., a compact, connected abelian Lie group. Let $X \in \mathfrak{t}=\operatorname{Lie}(T)$. For $X$ in the Lie algebra $\mathfrak{t}$ of $T$ (i.e., we view $X$ as a tangent vector at the identity element 1 to $T$ ), there exists a unique homomorphism $\alpha_{X}: \mathbb{R} \rightarrow T$ with $\alpha_{X}(0)=1, \alpha_{X}^{\prime}(0)=X$. Define the so-called exponential map exp: $\mathfrak{t} \rightarrow T$ by $\exp (X):=\alpha_{X}(1)$. Using the exponential map, one can generate many vector fields on a manifold from a given torus action. Indeed, for each $X \in \mathfrak{t}$, the vector field $\mathcal{G}(X)$ on $M$ generated by $T$-action from $X$ is defined by

$$
\mathcal{G}(X)_{p}:=\text { tangent vector to } \underbrace{t \mapsto \overbrace{\exp (t X)}^{\text {curve in } T} \cdot p}_{\text {curve in } M \text { through } p} \text { at } t=0 .
$$

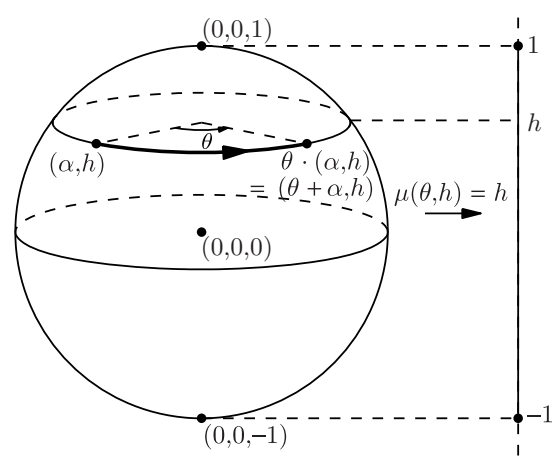

Figure 2.1. The momentum map for the 2 -sphere $S^{2}$ is the height function $\mu(\theta, h)=h$. The image of $S^{2}$ under the momentum map $\mu$ is the closed interval $[-1,1]$. Note that, as predicted by the Atiyah-Guillemin-Sternberg Theorem (see Theorem 2.2), the interval $[-1,1]$ is equal to the image under $\mu$ of the set $\{(0,0,-1),(0,0,1)\}$ of fixed points of the Hamiltonian $S^{1}$-action on $S^{2}$ by rotations about the vertical axis. 
A $T$-action on $(M, \omega)$ is symplectic if all the vector fields that it generates are symplectic, i.e., their flows preserve the symplectic form $\omega$. The T-action is Hamiltonian if all the vector fields it generates are Hamiltonian, i.e., they satisfy Hamilton's PDEs. Any symplectic action on a simply connected manifold is Hamiltonian.

From a given Hamiltonian torus action, one can construct a special type of map, which encodes information about the action - this is the famous momentum map. The construction of the momentum map is due to Kostant 62 and Souriau 95 (we refer to Marsden and Ratiu [76. pp. 369, 370] for the history of the momentum map). The momentum map can be defined with great generality for a Hamiltonian Lie group action. The momentum map was a key tool in Kostant's quantization lectures [63, and Souriau discussed it at length in his book 96]. Here we shall only deal with the momentum map in the rather particular case of torus actions, in which the construction is simpler.

Assume that $\operatorname{dim} T=m, \operatorname{dim} M=2 n$. Let $e_{1}, \ldots, e_{m}$ be a basis of the Lie algebra $\boldsymbol{t}$. Let $\mathcal{E}_{1}, \ldots, \mathcal{E}_{m}$ be the corresponding vector fields. By definition of Hamiltonian action, there exists a unique (up to a constant) Hamiltonian $H_{i}$ such that $\omega\left(\mathcal{E}_{i}, \cdot\right):=\mathrm{d} H_{i}$, i.e., $\mathcal{E}_{i}=\mathcal{H}_{H_{i}}$. Now we define the momentum map by

$$
\mu:=\left(H_{1}, \ldots, H_{m}\right): M \rightarrow \mathbb{R}^{m} .
$$

The map $\mu$ is unique up to composition by an element of $\operatorname{GL}(m, \mathbb{Z})$ (because our construction depends on the choice of a basis) and translations in $\mathbb{R}^{m}$ (because the Hamiltonians are defined only up to a constant).

The simplest example of a Hamiltonian torus action is given by $S^{2}$ with the rotational $S^{1}$-action depicted in Figure 2.1. It is easy to check that the momentum map for this action is the height function $\mu(\theta, h)=h$.

On the U.S. east coast the momentum map has traditionally been called "moment map", while on the west coast it has been traditional to use the term "momentum map". In French they both reconcile into the term "application moment".

2.3. Structure theorems for Hamiltonian actions. Much of the authors' intuition on integrable systems was originally guided by some remarkable results proved in the early 1980s by Atiyah, Guillemin and Sternberg, and Delzant, in the context of Hamiltonian torus actions. The first of these results was the following influential convexity theorem of Atiyah, and Guillemin and Sternberg.

Theorem 2.2 (Atiyah [6, Guillemin and Sternberg [56]). If an m-dimensional torus acts on a compact, connected $2 n$-dimensional symplectic manifold $(M, \omega)$ in a Hamiltonian fashion, then the image $\mu(M)$ of $M$ under the momentum map $\mu:=\left(H_{1}, \ldots, H_{m}\right): M \rightarrow \mathbb{R}^{m}$ is a convex polytope.

See Figures 2.1 and 2.2 for an illustration of the theorem. Other remarkable convexity theorems were proved after the theorem above by Kirwan 61] (in the case of compact, nonabelian group actions), Benoist 10] (in the case when the action is not necessarily Hamiltonian, but it has some coisotropic orbit) and Giacobbe [50]. Convexity in the case of Poisson actions has been studied by Alekseev [3], Flaschka and Ratiu [45, Ortega and Ratiu [82, and Weinstein [119] among others.

Recall that a convex polytope in $\mathbb{R}^{n}$ is simple if there are $n$ edges meeting at each vertex, rational if the edges meeting at each vertex have rational slopes, i.e., they are of the form $p+t u_{i}, 0 \leq t<\infty$, where $u_{i} \in \mathbb{Z}^{n}$, and smooth if the 

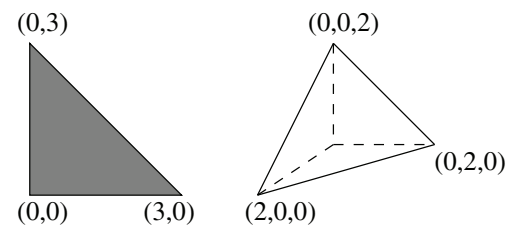

Figure 2.2. Delzant polytopes corresponding to the complex projective spaces $\mathbb{C P}^{2}$ and $\mathbb{C P}^{3}$ equipped with scalar multiples of the Fubini-Study symplectic form.

vectors $u_{1}, \ldots, u_{n}$ may be chosen to be a basis of $\mathbb{Z}^{n}$ (see Figure 2.2). In the mid1980s Delzant 30] showed the following classification result, which complements the Atiyah-Guillemin-Sternberg convexity theorem.

Theorem 2.3 (Delzant 30). If an $n$-dimensional torus acts effectively and Hamiltonianly on a compact, connected symplectic $2 n$-dimensional manifold $(M, \omega)$, the polytope in the Atiyah-Guillemin-Sternberg theorem is simple, rational, and smooth, and it determines the symplectic isomorphism type of $M$, and moreover, $M$ is a toric variety in the sense of complex algebraic geometry. Starting from any simple, rational smooth polytope $\Delta \subset \mathbb{R}^{m}$, one can construct a compact, connected symplectic manifold $\left(M_{\Delta}, \omega_{\Delta}\right)$ with an effective Hamiltonian action for which its associated polytope is $\Delta$.

By an isomorphism $\chi:\left(M_{1}, \omega_{1}\right) \rightarrow\left(M_{2}, \omega_{2}\right)$ in Theorem 2.3. we mean an equivariant symplectomorphism such that $\chi^{*} \mu_{2}=\mu_{1}$, where $\mu_{i}$ is the momentum map of $M_{i}, i=1,2$ (the map $\chi$ is an equivariant symplectomorphism in the sense that it is a diffeomorphism which pulls back the symplectic form $\omega_{2}$ to $\omega_{1}$ and commutes with the torus actions). The manifolds in Delzant's theorem are called symplectic-toric manifolds or Delzant manifolds. See Duistermaat and Pelayo [33. for a detailed study of the relation between Delzant manifolds and toric varieties in algebraic geometry. In the context of symplectic geometry, motivated by Delzant's results, one usually refers to simple, rational smooth polytopes as Delzant polytopes.

Delzant's theorem tells us that from the point of view of symplectic geometry, complex projective spaces endowed with the standard action by rotations of a torus, half the dimension of the corresponding complex projective space are simple polytopes. More precisely, consider the projective space $\mathbb{C P}^{n}$ equipped with a $\lambda$-multiple of the Fubini-Study form and the standard rotational action of $\mathbb{T}^{n}$ (for $\mathbb{C P}^{1}=S^{2}$, we already drew the momentum map in Figure 2.1). The complex projective space $\mathbb{C P}^{n}$ is a $2 n$-dimensional symplectic-toric manifold, and one can check that the momentum map is given by

$$
\mu(z)=\left(\frac{\lambda\left|z_{1}\right|^{2}}{\sum_{i=0}^{n}\left|z_{i}\right|^{2}}, \ldots, \frac{\lambda\left|z_{n}\right|^{2}}{\sum_{i=0}^{n}\left|z_{i}\right|^{2}}\right) .
$$

It follows that the momentum polytope equals the convex hull in $\mathbb{R}^{n}$ of 0 and the scaled canonical vectors $\lambda e_{1}, \ldots, \lambda e_{n}$; see Figure 2.2. Theorem 2.3 says that this polytope determines all the information about $\mathbb{C P}^{n}$, the symplectic form and the torus action. 
There have been many other contributions to the structure theory of Hamiltonian torus actions. Particularly worth noting is Karshon's paper [59] (see also [58]), where she gives a classification of Hamiltonian circle actions on compact connected 4-dimensional symplectic manifolds; we briefly review Karshon's result. To a compact, connected 4-dimensional symplectic manifold equipped with an effective Hamiltonian $S^{1}$-action (i.e., a so-called compact 4-dimensional Hamiltonian $S^{1}$-space), we may associate a labeled graph as follows. For each component $\Sigma$ of the set of fixed points of the $S^{1}$-action, there is one vertex in the graph, labeled by the real number $\mu(\Sigma)$, where $\mu: M \rightarrow \mathbb{R}$ is the momentum map of the action. If $\Sigma$ is a surface, then the corresponding vertex has two additional labels, one being the symplectic area of $\Sigma$, and the other one being the genus of $\Sigma$.

For every finite subgroup $F_{k}$ of $k$ elements of $S^{1}$ and for every connected component $C$ of the set of points fixed by $F_{k}$, we have an edge in the graph, labeled by the integer $k>1$. The component $C$ is a 2 -sphere, which we call a $F_{k}$-sphere. The quotient circle $S^{1} / F_{k}$ rotates it while fixing two points, and the two vertices in the graph corresponding to the two fixed points are connected in the graph by the edge corresponding to $C$.

On the other hand, it was proved by Audin, Ahara, and Hattori [2, 7, 8 that every compact 4-dimensional Hamiltonian $S^{1}$-space is isomorphic (meaning $S^{1}$ equivariantly diffeomorphic) to a complex surface with a holomorphic $S^{1}$-action which is obtained from $\mathbb{C P}^{2}$, a Hirzebruch surface or a $\mathbb{C P}^{1}$-bundle over a Riemann surface (with appropriate circle actions), by a sequence of blow-ups at the fixed points.

Let $A$ and $B$ be connected components of the set of fixed points. The $S^{1}$-action extends to a holomorphic action of the group $\mathbb{C}^{\times}$of nonzero complex numbers. Consider the time flow given by the action of subgroup $\exp (t), t \in \mathbb{R}$. We say that $A$ is greater than $B$ if there is an orbit of the $\mathbb{C}^{\times}$-action which at time $t=\infty$ approaches a point in $A$ and at time $t=-\infty$ approaches a point in $B$.

Take any of the complex surfaces with $S^{1}$-actions considered by Audin, Ahara, and Hattori, and assign a real parameter to every connected component of the set of fixed points such that these parameters are monotonic with respect to the partial ordering we have just described. If the manifold contains two fixed surfaces, then assign a positive real number to each of them in such a way that the difference between the numbers is given by a formula involving the previously chosen parameters. Karshon proved [58, Theorem 3] that for every such choice of parameters there exists an invariant symplectic form and a momentum map on the complex surface such that the values of the momentum map at the fixed points and the symplectic areas of the fixed surfaces are equal to the chosen parameters. Moreover, every two symplectic forms with this property differ by an $S^{1}$-equivariant diffeomorphism. Karshon proved the following classification result à la Delzant.

Theorem 2.4 (Karshon [59]). If two compact Hamiltonian $S^{1}$-space.5 have the same graph, then they are isomorphic (i.e., $S^{1}$-equivariantly symplectomorphic). Moreover, every compact 4-dimensional Hamiltonian $S^{1}$-space is isomorphic to one of the spaces listed in the paragraph above.

${ }^{5}$ That is, two compact, connected 4-dimensional manifolds equipped with an effective Hamiltonian $S^{1}$-action. 
Again, in Theorem 2.4. an isomorphism is an equivariant symplectomorphism which pulls back the momentum map on one manifold to the momentum map on the other manifold. Theorem 2.4 has useful consequences; for example, every compact Hamiltonian $S^{1}$-space admits an $S^{1}$-invariant complex structure for which the symplectic form is Kähler.

2.4. Structure theorems for symplectic actions. From the viewpoint of symplectic geometry, the situation described by the momentum polytope is very rigid. It is natural to wonder whether the structure results Theorem 2.2 and Theorem 2.3 for Hamiltonian actions of tori persist in a more general context. There are at least two natural ways to approach this question, which we explain next.

First, one can insist on having a compact group action, but not require that the group acts in a Hamiltonian fashion. In other words, do the striking theorems above persist if the vector fields generated by action have flows that preserve symplectic form (i.e., are symplectic) but Hamilton's PDEs have no solution (i.e., the vector fields are not Hamiltonian)? Many easy examples fit this criterion: for example, take the 2-torus $\mathbb{T}^{2}$ with the standard area form $\mathrm{d} \theta \wedge \mathrm{d} \alpha$ and with the $\mathbb{S}^{1}$-action on the $\theta$-component; the basic vector field $\frac{\partial}{\partial \theta}$ is symplectic, but one can easily check that it is non-Hamiltonian.

Various works by Giacobbe [50, Benoist [10, Ortega and Ratiu [81, Duistermaat and Pelayo 32, and Pelayo 83 follow this direction. Benoist's paper gives a convexity result for symplectic manifolds with coisotropic orbits; Ortega and Ratiu give a general symplectic local normal form theorem, also studied by Benoist in the case that the orbits are coisotropic. The papers by Duistermaat and Pelayo provide classifications à la Delzant. Let us briefly recall these classifications.

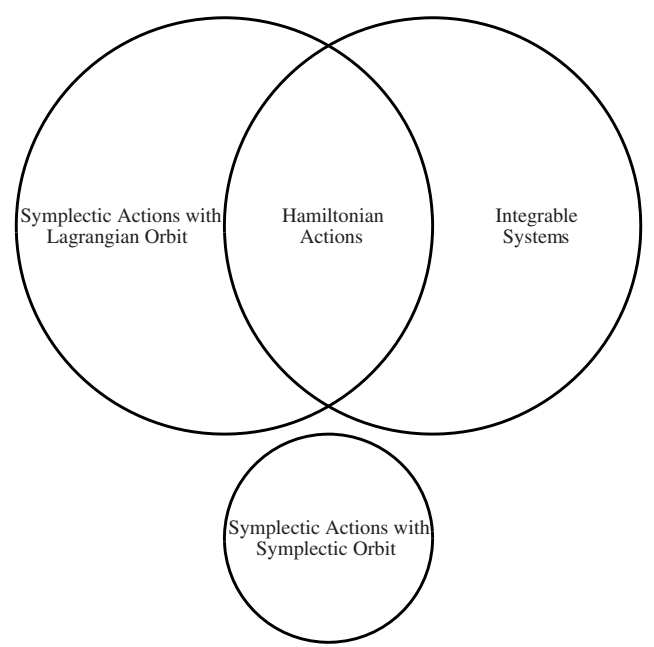

FiguRE 2.3. Hamiltonian $n$-torus actions may be viewed as a subclass of completely integrable Hamiltonian systems on $2 n$ dimensional manifolds, which we study later in this paper, and as a subclass of general symplectic actions, which have some Lagrangian orbit. 
Remark 2.5. Hamiltonian torus actions on compact manifolds always have fixed points (equivalently, the Hamiltonian vector fields generated by Hamiltonian torus actions always have fixed points). Sometimes the condition of being Hamiltonian for vector fields can be detected from the existence of fixed points; this is, in general, a challenging question.

The first result concerning the relationship between the existence of fixed points and the Hamiltonian character of vector fields generated by a $G$-action is Frankel's celebrated theorem [47] which says that if the manifold is compact, connected, and Kähler, $G=S^{1}$, and if the symplectic action has fixed points, then it must be Hamiltonian. Frankel's influential work has inspired subsequent research. McDuff [79. Proposition 2] has shown that any symplectic circle action on a compact, connected, symplectic 4-manifold having fixed points is Hamiltonian. See Tolman and Weitsman [102, Theorem 1], Feldman [48, Theorem 1], 65, Section 8], 74, Giacobbe [50, Theorem 3.13], Duistermaat and Pelayo [32, Corollary 3.9], Ginzburg [51, Proposition 4.2], Pelayo and Tolman [86 for additional results in the case of compact manifolds, and Pelayo and Ratiu 84 for results in the case of noncompact manifolds.

Our next goal is to present a classification à la Delzant of symplectic torus actions that have some Lagrangian orbit; this in particular includes all symplectic toric manifolds, because the maximal (in the sense of dimension) orbits of a symplectictoric manifold are Lagrangian. An $n$-dimensional submanifold $L$ of a symplectic $2 n$-manifold $(M, \omega)$ is Lagrangian if the symplectic form $\omega$ vanishes on $L$. For example, the orbits of the $S^{1}$-action by rotations on $S^{2}$ in Figure 2.1 are Lagrangian because an orbit is given by $h=$ const for some constant and the symplectic form is $\mathrm{d} \theta \wedge \mathrm{d} h$, which clearly vanishes when $h$ is constant. So the maximal orbits of the standard symplectic 2-sphere are Lagrangian.

A famous example of a symplectic manifold with a 2-torus action for which all the orbits are Lagrangian is the the Kodaira-Thurston manifold. It is constructed as follows. Let $\left(j_{1}, j_{2}\right) \in \mathbb{Z}^{2}$ act on $\mathbb{R}^{2}$ by the inclusion map (i.e., $\left(j_{1}, j_{2}\right) \cdot\left(x_{1}, y_{1}\right)=$ $\left.\left(j_{1}+x_{1}, j_{2}+y_{1}\right)\right)$, on $\mathbb{T}^{2}$ by the 2 -by- 2 matrix with entries $a_{11}=a_{22}=1, a_{12}=j_{2}$, $a_{21}=0$, and on the product $\mathbb{R}^{2} \times \mathbb{T}^{2}$ by the diagonal action. This diagonal action gives rise to a torus bundle over a torus $\mathbb{R}^{2} \times \mathbb{Z}^{2} \mathbb{T}^{2}$, the total space of which is compact and connected. The product symplectic form $\mathrm{d} x_{1} \wedge \mathrm{d} y_{1}+\mathrm{d} x_{2} \wedge \mathrm{d} y_{2}$ on $\mathbb{R}^{2} \times \mathbb{Z}^{2}$ descends to a symplectic form on $\mathbb{R}^{2} \times \mathbb{Z}^{2} \mathbb{T}^{2}$.

Moreover, one can check that $\mathbb{T}^{2}$ acts symplectically on $\mathbb{R}^{2} \times_{\mathbb{Z}^{2}} \mathbb{T}^{2}$, where the first circle of $\mathbb{T}^{2}$ acts on the left-most component of $\mathbb{R}^{2}$, and the second circle acts on the right-most component of $\mathbb{T}^{2}$ (one can check that this is indeed a well-defined, free symplectic action). Because the action is free, it does not have fixed points, and hence it is not Hamiltonian (it follows from the Atiyah-Guillemin-Sternberg theorem that Hamiltonian actions always have some fixed point). All the orbits of this action are Lagrangian submanifolds, because both factors of the symplectic form vanish since each factor has a component which is zero because it is the differential of a constant.

Another example is $\mathbb{T}^{2} \times S^{2}$ equipped with the form $\mathrm{d} x \wedge \mathrm{d} y+\mathrm{d} \theta \wedge \mathrm{d} h$, on which the 2 -torus $\mathbb{T}^{2}$ acts symplectically, one circle on each factor. This action has no fixed points, so it is not Hamiltonian. It is also not free. The maximal orbits are Lagrangian. All of these examples fit in the following theorem. 
Theorem 2.6 (Duistermaat and Pelayo [32]). Assume that a torus $\mathbb{T}^{m}$ of dimension $m$ acts effectively and symplectically on a compact, connected, symplectic $2 m$ manifold $(M, \omega)$ with some Lagrangian orbit. Then $\mathbb{T}^{m}$ decomposes as a product of two subtori $\mathbb{T}^{m}=T_{\mathrm{h}} T_{\mathrm{f}}$, where $T_{\mathrm{h}}$ acts Hamiltonianly on $M$ and $T_{\mathrm{f}}$ acts freely on $M$, and there is two-step fibration $M \rightarrow X \rightarrow S$, where $M$ is the total space of a fibration over $X$ with fibers symplectic-toric manifolds $\left(M_{\mathrm{h}}, T_{\mathrm{h}}\right)$, and $X$ is a $T_{\mathrm{f}}$-bundle over a torus $S$ of dimension $m-\operatorname{dim} T_{\mathrm{h}}$.

In this theorem $X$ is a symplectic homogenous space for the twisted group $T \times \mathfrak{t}^{*}$. The formulation of this theorem in 32 is completely explicit, but it is too involved to be described here. In particular, the formulation contains a complete symplectic classification in terms of six symplectic invariants (e.g., the Chern class of the fibration, the Hamiltonian torus $T_{\mathrm{h}}$, the polytope corresponding to the Hamiltonian action of $T_{\mathrm{h}}$, etc.) This classification includes Delzant's classification (stated previously in the paper as Theorem 2.3), which corresponds to the case of $T_{\mathrm{h}}=\mathbb{T}^{m}$ and $T_{\mathrm{f}}$ is trivial; in this case, five of the invariants do not appear, the only invariant is the polytope. Note that the "opposite" situation occurs when $T_{\mathrm{f}}=\mathbb{T}^{m}$ and $T_{\mathrm{h}}$ is trivial (e.g., the Kodaira-Thurston manifold), and in this case Theorem 2.6 says that $M$ is a torus bundle over a torus with Lagrangian fibers. An example of a manifold which fits in Theorem 2.6 is the family of 10-dimensional twisted examples with Lagrangian orbits which is illustrated in Figure 2.4.

A classification theorem in the case when there exists a maximal symplectic orbit (i.e., an orbit on which the symplectic form is restricted to a symplectic form) was proved in 83 . In the same paper, a classification of symplectic actions of 2-tori on

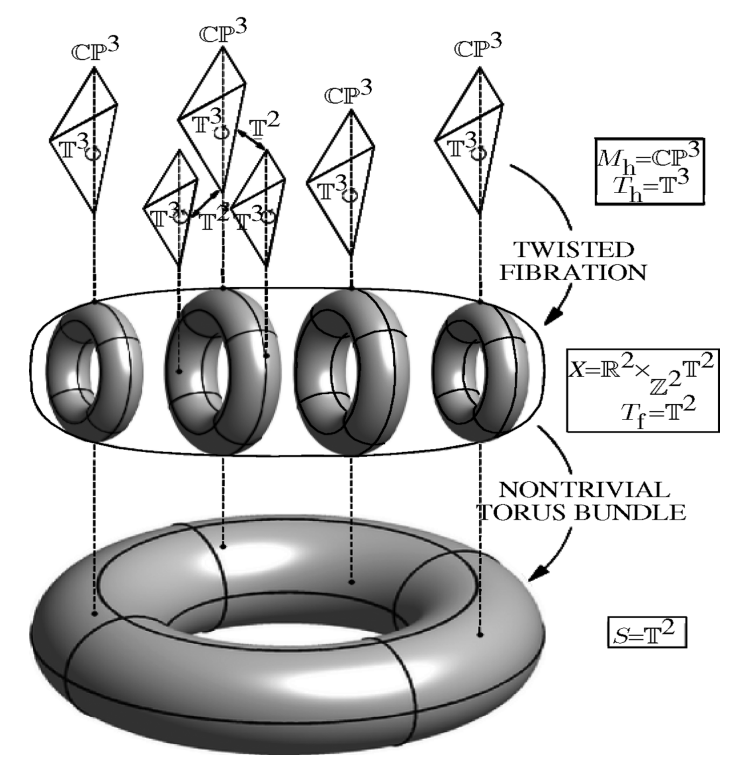

FiguRE 2.4. 10-dimensional symplectic manifolds with a torus action with Lagrangian orbits. The vector field generated by $T$-action is a "twist" of $\mathcal{Y}=\left(\mathcal{Y}_{\mathrm{h}}, \mathcal{Y}_{\mathrm{f}}\right)$, where $\mathcal{Y}_{\mathrm{h}}$ is Hamiltonian on $\mathbb{C P}^{3}$ and $\mathcal{Y}_{\mathrm{f}}$ is symplectic on $\mathbb{R}^{2} \times_{\mathbb{Z}^{2}} \mathbb{T}^{2}$. 
compact connected symplectic 4-manifolds was given that builds on this result and Theorem 2.6.

Theorem 2.7 (Pelayo [83, Duistermaat and Pelayo [34]). A compact connected symplectic 4-manifold $(M, \omega)$ equipped with an effective symplectic action of a 2torus is isomorphic (i.e., equivariantly symplectomorphic) to one, and only one, of the spaces in the table:

\begin{tabular}{|ccc|ccc|}
\hline SPACE & ACTION & $\begin{array}{c}\text { MAXIMAL } \\
\text { ORBIts }\end{array}$ & HAMILTONIAN? & $\begin{array}{c}\text { INVARIANT } \\
\text { COMPLEX? }\end{array}$ & KÄHLER? \\
\hline Toric & Fixed points & Lagrangian & Yes & Yes & Yes \\
\hline$M \rightarrow \Sigma$ & Locally Free & Symplectic & No & Yes & Yes \\
\hline$M \rightarrow \mathbb{T}^{2}$ & Free & Lagrangian & No & Yes & No \\
\hline $\mathbb{T}^{2} \times S^{2}$ & Else & Lagrangian & No & Yes & Yes \\
\hline
\end{tabular}

The first item is a symplectic-toric manifold with its standard Hamiltonian 2-torus action. The second item is an orbifold 2-torus bundle over a 2-dimensional compact connected orbifold $\Sigma$. The third item is a 2-torus bundle over a 2-torus.

Remark 2.8. The study of the existence of complex (and Kähler) structures on manifolds with symplectic torus actions appears to be a challenging question in dimensions greater than 4 . We do not know at this time how to approach this problem (which is fully answered in dimension 4 in Theorem 2.7) for the manifolds in Theorem 2.6. We do know, however, that many of these manifolds are Kähler, and may are not; see Lin and Pelayo 72$]$.

The classification in the first four columns in the table in Theorem 2.7 was the main result of [83, and the last two columns were proved in Duistermaat and Pelayo 32] the article [32] is based on Kodaira's seminal work [66. Theorem 19] of 1961 on complex analytic surfaces. Moreover, the two middle items in the table above are completely explicit and classified in terms of five symplectic invariants; cf. 83, Theorem 8.2.1].

Example 2.9. Let us spell out the space on the third row in the table given in Theorem 2.7 more concretely, and we refer to Pelayo [83, Section 8] for the construction of the second row. The construction which we present next is selfcontained and provides a source of many inequivalent examples. Let $T$ be a 2 dimensional torus. Let $T_{\mathbb{Z}}$ be the kernel of the exponential mapping $\exp : \mathfrak{t} \rightarrow T$.

a) For any choice of

i) a discrete cocompact subgroup $P$ of $\mathfrak{t}^{*}$, and

ii) a nonzero antisymmetric bilinear mapping $c: \mathfrak{t}^{*} \times \mathfrak{t}^{*} \rightarrow \mathfrak{t}$ such that $c(P \times P) \subset T_{\mathbb{Z}}$,

let $\iota: P \rightarrow T \times \mathfrak{t}^{*}$ be given by $\zeta=\zeta_{1} \epsilon_{1}+\zeta_{2} \epsilon_{2} \mapsto\left(\mathrm{e}^{-1 / 2 \zeta_{1} \zeta_{2} c\left(\epsilon_{1}, \epsilon_{2}\right)}, \zeta\right)$, where $\epsilon_{1}, \epsilon_{2}$ is a $\mathbb{Z}$-basis of $P$. The mapping $\iota$ is a homomorphism onto a discrete cocompact subgroup of $T \times \mathfrak{t}^{*}$ with respect to the nonstandard standard group structure given by

$$
(t, \zeta)\left(t^{\prime}, \zeta^{\prime}\right)=\left(t t^{\prime} \mathrm{e}^{-c\left(\zeta, \zeta^{\prime}\right) / 2}, \zeta+\zeta^{\prime}\right) .
$$

Equip $T \times \mathfrak{t}^{*}$ with the standard cotangent bundle symplectic form. Equip $\left(T \times \mathfrak{t}^{*}\right) / \iota(P)$ with the action of $T$ which comes from the action of $T$ by 
translations on the left factor of $T \times \mathfrak{t}^{*}$ and with the symplectic form induced by the symplectic form on $T \times \mathfrak{t}^{*}$. Then $\left(T \times \mathfrak{t}^{*}\right) / \iota(P)$ is a compact, connected symplectic 4-manifold on which $T$ acts freely and for which the $T$-orbits are Lagrangian 2-tori. Theorem 2.7 implies that two symplectic manifolds constructed in this way are isomorphic (i.e., $T$-equivariantly symplectomorphic) if and only if the corresponding cocompact subgroups and the corresponding bilinlear forms are equal.

b) For any choice of

i) a discrete cocompact subgroup $P$ of $\mathfrak{t}^{*}$, and

ii) a homomorphism $\tau: P \rightarrow T, \zeta \mapsto \tau_{\zeta}$,

let $\iota: P \rightarrow T \times \mathfrak{t}^{*}$ be given by $\zeta \mapsto\left(\tau_{\zeta}^{-1}, \zeta\right)$. The mapping $\iota$ is a homomorphism onto a discrete cocompact subgroup of $T \times \mathfrak{t}^{*}$ with respect to the standard group structure. Equip $T \times \mathfrak{t}^{*}$ with the standard cotangent bundle symplectic form. Then $\left(T \times \mathfrak{t}^{*}\right) / \iota(P)$ equipped with the induced $T$-action and symplectic form is a compact, connected symplectic 4-manifold on which $T$ acts freely with $T$-orbits Lagrangian 2-tori. Theorem 2.7 implies that two symplectic manifolds constructed in this way are isomorphic if and only if the corresponding cocompact groups $P$ and the corresponding equivalence classes $\tau \cdot \exp \left(\left.\operatorname{Sym}\right|_{P}\right) \in \mathcal{T}$ are equal. Here exp: $\operatorname{Hom}(P, \mathfrak{t}) \rightarrow \operatorname{Hom}(P, T)$ is the exponential map of the Lie group $\operatorname{Hom}(P, T)$ and $\left.\operatorname{Sym}\right|_{P} \subset \operatorname{Hom}(P, \mathfrak{t})$ is the space of restrictions $\left.\alpha\right|_{P}$ of linear maps $\alpha: \mathfrak{t}^{*} \rightarrow \mathfrak{t}, \xi \mapsto \alpha_{\xi}$, which are symmetric in the sense that for all $\xi, \xi^{\prime} \in \mathfrak{t}^{*}, \xi\left(\alpha_{\xi^{\prime}}\right)-\xi^{\prime}\left(\alpha_{\xi}\right)=0$.

In both cases above the projection mapping $\left(T \times \mathfrak{t}^{*}\right) / \iota(P) \rightarrow \mathfrak{t}^{*} / P$ is a principal $T$-bundle over the torus $\mathfrak{t}^{*} / P$ with Lagrangian fibers (the $T$-orbits). These spaces $\left(T \times \mathfrak{t}^{*}\right) / \iota(P)$ are all the possible cases that can occur as the third item in Theorem 2.7 .

Another natural generalization of a Hamiltonian torus action is the notion of a completely integrable system, or more generally, of a Hamiltonian system. Probably the most fundamental difference between the theory of Hamiltonian torus actions on compact manifolds and the theory of completely integrable Hamiltonian systems can be seen already at a local level. Completely integrable systems have, in general, singularities that are quite difficult to understand from a topological, dynamical, and analytic viewpoint. The singularities of Hamiltonian torus actions occur at the lower-dimensional orbits only and are tori of varying dimensions, but the case of integrable systems will usually exhibit a wider range of singularities, such as pinched tori; e.g., see Figure 3.1. Indeed, it is only recently that we are beginning to understand some of these singularities in low dimensions. The rest of this paper is focused on the local and global aspects of the symplectic geometry of completely integrable systems.

\section{Completely integrable systems}

3.1. Hamiltonian integrable systems. Let $(M, \omega)$ be a $2 n$-dimensional symplectic manifold. The pair consisting of the smooth manifold and a classical observable $H: M \rightarrow \mathbb{R}$ in $\mathrm{C}^{\infty}(M)$ is called a Hamiltonian system.

A famous example of a Hamiltonian system is the spherical pendulum, which is mathematically described as the symplectic cotangent bundle $\left(\mathrm{T}^{*} S^{2}, \omega_{\mathrm{T}^{*} S^{2}}\right)$ of the 
unit sphere equipped with the Hamiltonian

$$
H(\underbrace{\theta, \varphi}_{\text {sphere }}, \underbrace{\xi_{\theta}, \xi_{\varphi}}_{\text {fiber }})=\underbrace{\frac{1}{2}\left(\xi_{\theta}^{2}+\frac{1}{\sin ^{2} \theta} \xi_{\varphi}^{2}\right)}_{\text {kinetic energy }}+\underbrace{\cos \theta}_{\text {potential }} .
$$

Here $(\theta, \varphi)$ are the standard spherical angles ( $\varphi$ stands for the rotation angle around the vertical axis, while $\theta$ measures the angle from the north pole), and $\left(\xi_{\theta}, \xi_{\varphi}\right)$ are the cotangent conjugate variables. The function $H$ is smooth on $T^{*} S^{2}$ (the apparent singularity $1 / \sin ^{2} \theta$ is an artifact of the spherical coordinates).

A classical observable $H$ gives rise to the Hamiltonian vector field $\mathcal{H}_{H}$ on $M$, which is defined uniquely by $\omega\left(\mathcal{H}_{H}, \cdot\right)=\mathrm{d} H$. The algebra $\mathrm{C}^{\infty}(M)$ of classical observables, which we have been calling Hamiltonians, comes naturally endowed with the Poisson bracket, $\{J, H\}:=\omega\left(\mathcal{H}_{J}, \mathcal{H}_{H}\right)$. As a derivation, $\mathcal{H}_{H}$ is just the Poisson bracket by $H$; in other words, the evolution of a function $f$ under the flow of $\mathcal{H}_{H}$ is given by the equation $\dot{f}=\{H, f\}$.

An integral of the Hamiltonian $H$ is a function that is invariant under the flow of $\mathcal{H}_{H}$, i.e., a function $f$ such that $\{H, f\}=0$. The Hamiltonian $H$ is said to be completely integrable if there exists $n-1$ independent functions $f_{2}, \ldots, f_{n}$ (independent in the sense that the differentials $\mathrm{d}_{m} H, \mathrm{~d}_{m} f_{2}, \ldots, \mathrm{d}_{m} f_{n}$ are linearly independent at almost every point $m \in M$ ) that are integrals of $H$ and that pairwise Poisson-commute, i.e., $\left\{H, f_{i}\right\}=0$ and $\left\{f_{i}, f_{j}\right\}=0$. For example, the spherical pendulum $\left(\mathrm{T}^{*} S^{2}, \omega_{\mathrm{T}^{*} S^{2}}, H\right)$ is integrable by considering the vertical angular momentum, which is the function $f_{2}\left(\theta, \varphi, \xi_{\theta}, \xi_{\varphi}\right)=\xi_{\varphi}$. In the abstract definition of a completely integrable system, it is clear that $H$ does not play a distinguished role among the functions $f_{2}, \ldots, f_{n}$. The point of view in this paper will always be to consider, as a whole, a collection of such functions. The integer $n$ is traditionally called the number of degrees of freedom of the system.

Definition 3.1. A completely integrable system on the $2 n$-dimensional symplectic manifold $M$, compact or not, is a collection of $n$ Poisson commuting functions $f_{1}, \ldots, f_{n} \in \mathrm{C}^{\infty}(M)$ which are independent.

An important class of completely integrable systems are those given by Hamiltonian $n$-torus actions on symplectic $2 n$-manifolds (i.e., symplectic-toric manifolds). These actions have a momentum map with $n$ components $f_{1}, \ldots, f_{n}$, and these components always form a completely integrable system in the sense of the definition above.

3.2. Singularities and regular points. From a topological, analytical, and dynamical viewpoint, the most interesting features of the completely integrable system on a symplectic manifold are encoded in the "singular" fibers of the momentum map $F=\left(f_{1}, \ldots, f_{n}\right): M \rightarrow \mathbb{R}^{n}$, and in their surrounding neighborhoods. We frequently refer to $F$ itself as the integrable system.

A point $m \in M$ is called a regular point if $\mathrm{d}_{m} F$ has rank $n$. A point $c \in \mathbb{R}^{n}$ is a regular value if the fiber $F^{-1}(c)$ contains only regular points. If $c$ is a regular value, the fiber $F^{-1}(c)$ is called a regular fiber. A point $m \in M$ is a critical point, or a singularity, if $\mathrm{d}_{m} F$ has rank strictly less than $n$. Geometrically, this means that the Hamiltonian vector fields generated by the components of $F$ are linearly dependent at $m$; see Figure 3.1. A fiber $F^{-1}(c)$ is a singular fiber if it contains at least one critical point; see Figure 5.4 . 

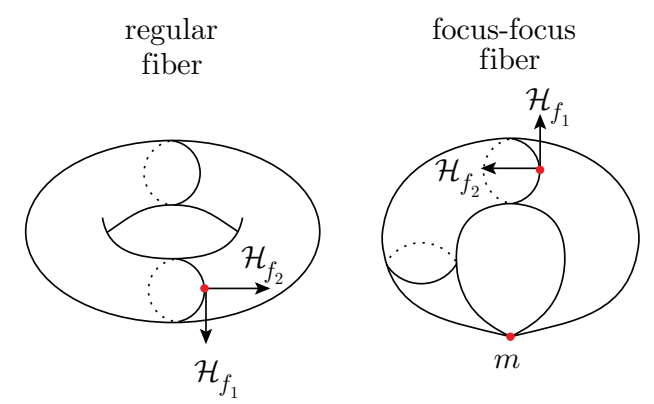
transversally
elliptic fiber

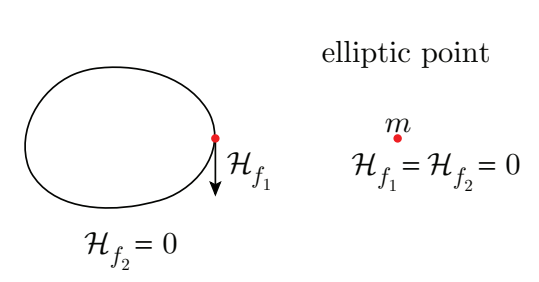

FiguRE 3.1. The figures show some possible singularities of a completely integrable system. In the left-most figure, $m$ is a regular point (rank 2). In the second figure $m$ is a focus-focus point (rank 0 ). In the third one, $m$ is a transversally elliptic singularity (rank 1). In the right-most figure, $m$ is an elliptic-elliptic point.

It follows from the definition of a completely integrable system (simply follow the flows of the Hamiltonian vector fields) that if $X$ is a connected component of a regular fiber $F^{-1}(c)$, and if the vector fields $\mathcal{H}_{f_{1}}, \ldots, \mathcal{H}_{f_{n}}$ are complete on $F^{-1}(c)$, then $X$ is diffeomorphic to $\mathbb{R}^{n-k} \times \mathbb{T}^{k}$. Moreover, if the regular fiber $F^{-1}(c)$ is compact, then $\mathcal{H}_{f_{1}}, \ldots, \mathcal{H}_{f_{n}}$ are complete, and thus the component $X$ is diffeomorphic to $\mathbb{T}^{n}$; this is always true if, for example, some component $f_{i}$ is proper.

The study of singularities of integrable systems is fundamental for various reasons. On the one hand, because of the way an integrable system is defined in terms $n$ smooth functions on a manifold, it is expected (apart from exceptional cases) that singularities will necessarily occur. On the other hand these functions define a dynamical system such that their singularities correspond to fixed points and relative equilibria of the system, which are of course one of the main characteristics of the dynamics.

As a remark for those interested in semiclassical analysis, we note that from a semiclassical viewpoint, we know furthermore that important wave functions such as eigenfunctions of the quantized system have a microsupport which is invariant under classical dynamics; therefore, in a sense that we shall not present here (one should talk about semiclassical measures), they concentrate near certain singularities6 (see for instance [22] and the work of Toth [103]). This concentration entails not only the growth in norm of eigenfunctions (see for instance [104]) but also a higher local density of eigenvalues 23, 106, 26]).

Let $f_{1}, \ldots, f_{n}$ define an integrable system on a symplectic manifold $M$, and let $F=\left(f_{1}, \ldots, f_{n}\right)$ be the associated momentum map. Suppose that $F$ is a proper map so that the regular fibers of $F$ are $n$-dimensional tori. Liouville proved in 1855 73 that, locally, the equations of motion defined by any of the functions $f_{i}$ are integrable by quadratures. This holds in a neighborhood of any point where the differentials $\mathrm{d} f_{j}$ are linearly independent.

A pleasant formulation of Liouville's result, due to Darboux and Carathéodory, says that there exist canonical coordinates $(x, \xi)$ in which the functions $f_{j}$ are

\footnotetext{
${ }^{6}$ Those called hyperbolic.
} 
merely the "momentum coordinates" $\xi_{j}$. In 1935 Henri Mineur 75] stated 7 in the special case of $\mathbb{R}^{n} \times \mathbb{R}^{n}$ that if $\Lambda$ is a compact level set of the momentum map $F$, then $\Lambda$ is a torus. Moreover, there exist symplectic coordinates $(x, \xi)$, where $x$ varies in the torus $\mathbb{T}^{n}=\mathbb{R}^{n} / \mathbb{Z}^{n}$ and $\xi$ varies in a neighborhood of the origin in $\mathbb{R}^{n}$, in which the functions $f_{j}$ depend only on the $\xi$-variables. In geometric terms, the system is symplectically equivalent to a neighborhood of the zero section of the cotangent bundle $\mathrm{T}^{*}\left(\mathbb{T}^{n}\right)$ equipped with the integrable system $\left(\xi_{1}, \ldots, \xi_{n}\right)$. This result, proved in the general case in 1963 by Arnold 4] (Arnold was not aware of Mineur's work), is known as the action-angle theorem or the Liouville-Arnold theorem. The tori are the famous Liouville tori. Although Liouiville's theorem has been originally attached to this theorem, we are not aware of Liouville having contributed to this result; we thank J. J. Duistermaat for pointing this out to us [36.

We will study these and other results in more detail in the following two sections.

\section{LOCAL THEORY OF COMPLETELY INTEGRABLE SYSTEMS}

4.1. Local model at regular points. Let $\left(f_{1}, \ldots, f_{n}\right)$ be a completely integrable system on a $2 n$-symplectic manifold $M$ with momentum map $F$. By the local submersion theorem, the fibers $F^{-1}(c)$ for $c$ close to $F(m)$ are locally $n$-dimensional submanifolds near a regular point $m$. The local structure of regular points of completely integrable systems is simple:

Theorem 4.1 (Darboux-Carathéodory). Let $\left(f_{1}, \ldots, f_{n}\right)$ be a completely integrable system on a $2 n$-symplectic manifold $M$, with momentum map $F$. If $m$ is regular, $F$ is symplectically conjugate near $m$ to the linear fibration $\left(\xi_{1}, \ldots, \xi_{n}\right)$ on the symplectic space $\mathbb{R}^{2 n}$ with coordinates $\left(x_{1}, \ldots, x_{n}, \xi_{1}, \ldots, \xi_{n}\right)$ and symplectic form $\sum_{i} \mathrm{~d} \xi_{i} \wedge \mathrm{d} x_{i}$

In other words, the Darboux-Carathéodory theorem says that there exists smooth functions $\phi_{1}, \ldots, \phi_{n}$ on $M$ such that $\left(\phi_{1}, \ldots, \phi_{n}, f_{1}, \ldots, f_{n}\right)$ is a system of canonical coordinates in a neighborhood of $m$. In principle the name of Liouville should be associated with this theorem, since well before Darboux and Carathéodory, Liouville gave a nice explicit formula for the functions $\phi_{j}$. This result, published in 1855 [73, explains the local integration of the flow of any completely integrable Hamiltonian (possibly depending on time) near a regular point of the foliation in terms of the Liouville 1-form $\sum_{i} \xi_{i} \mathrm{~d} x_{i}$. In this respect it implies the Darboux-Carathéodory theorem, even if Liouville's formulation is more complicated.

4.2. Local models at singular points. One can approach the study of the singularities of Hamiltonian systems in two different ways: one can analyze the flow of the vector fields - this is the "dynamical systems" viewpoint - or one can study of the Hamiltonian functions themselves - this is the "foliation" perspective.

In the case of completely integrable systems, the dynamical and foliation points of view are equivalent because the vector fields of the $n$ functions $f_{1}, \ldots, f_{n}$ form a basis of the tangent spaces of the leaves of the foliation $f_{i}=$ const $_{i}$, at least for regular points. The foliation perspective usually displays better the geometry of the problem, and we will frequently use this viewpoint. However, the foliations

${ }^{7}$ J. J. Duistermaat pointed out some gaps in Mineur's proof; see 36. 
we are interested in are singular, and the notion of a singular foliation is already delicate. Generally speaking, these foliations are of Stefan-Süßmann type [97]: the leaves are defined by an integrable distribution of vector fields. But they are more than that: they are Hamiltonian, and they are almost regular in the sense that the singular leaves cannot fill up a domain of positive measure.

4.2.1. nondegenerate critical points. In singularity theory for differentiable functions, "generic" singularities are Morse singularities. In the theory of completely integrable systems there exists a natural analogue of the notion of Morse singularities (or more generally of Morse-Bott singularities if one allows critical submanifolds). These so-called nondegenerate singularities are now well defined and exemplified in the literature, so we will only recall briefly the definition in Vey's paper [105.

Let $F=\left(f_{1}, \ldots, f_{n}\right)$ be a completely integrable system on $M$. A fixed point $m \in M$ is called nondegenerate if the Hessians $\mathrm{d}_{m}^{2} f_{j}$ span a Cartan subalgebra of the Lie algebra of quadratic forms on the tangent space $\mathrm{T}_{m} M$ equipped with the linearized Poisson bracket. This definition applies to a fixed point; more generally, if $\mathrm{d}_{m} F$ has corank $r$, one can assume that the differentials $\mathrm{d}_{m} f_{1}, \ldots, \mathrm{d}_{m} f_{n-r}$ are linearly independent; then we consider the restriction of $f_{n-r+1}, \ldots, f_{n}$ to the symplectic manifold $\Sigma$ obtained by local symplectic reduction under the action of $f_{1}, \ldots, f_{n-r}$. We shall say that $m$ is nondegenerate (or transversally nondegenerate) whenever $m$ is a nondegenerate fixed point for this restriction of the system to $\Sigma$.

In order to understand the following theorem one has to know the linear classification of Cartan subalgebras of $\operatorname{sp}(2 n, \mathbb{R})$. This follows from the work of Williamson 113, which shows that any such Cartan subalgebra has a basis built with three types of blocks: two unidimensional ones (the elliptic block $q=x^{2}+\xi^{2}$, and the real hyperbolic one $q=x \xi$ ) and a two-dimensional block called focus-focus: $q_{1}=x \eta-y \xi, q_{2}=x \xi+y \eta$. If $k_{\mathrm{e}}, k_{\mathrm{h}}, k_{\mathrm{f}}$, respectively, denote the number of elliptic, hyperbolic and focus-focus components, we may associate the triple $\left(k_{\mathrm{e}}, k_{\mathrm{h}}, k_{\mathrm{f}}\right)$ to a singularity. The triple is called by Nguyên Tiên Zung the Williamson type of the singularity.

Theorem 4.2 (Eliasson [2, 43, 112]). The nondegenerate critical points of a completely integrable system are linearizable. That is, if $m \in M$ is a nondegenerate critical point of the completely integrable system $F=\left(f_{1}, \ldots, f_{n}\right): M \rightarrow \mathbb{R}^{n}$, then there exist local symplectic coordinates $\left(x_{1}, \ldots, x_{n}, \xi_{1}, \ldots, \xi_{n}\right)$ about $m$, in which $m$ is represented as $(0, \ldots, 0)$ and such that $\left\{f_{i}, q_{j}\right\}=0$, for all indices $i, j$, where we have the following possibilities for the components $q_{1}, \ldots, q_{n}$, each of which is defined on a small neighborhood of $(0, \ldots, 0)$ in $\mathbb{R}^{n}$ :

(i) Elliptic component: $q_{j}=\left(x_{j}^{2}+\xi_{j}^{2}\right) / 2$, where $j$ may take any value $1 \leq j \leq n$.

(ii) Hyperbolic component: $q_{j}=x_{j} \xi_{j}$, where $j$ may take any value $1 \leq j \leq n$.

(iii) Focus-focus component: $q_{j-1}=x_{j-1} \xi_{j}-x_{j} \xi_{j-1}$ and $q_{j}=x_{j-1} \xi_{j-1}+x_{j} \xi_{j}$, where $j$ may take any value $2 \leq j \leq n-1$ (note that this component appears as "pairs").

(iv) Nonsingular component: $q_{j}=\xi_{j}$, where $j$ may take any value $1 \leq j \leq n$.

Moreover, if $m$ does not have any hyperbolic block, then the system of commuting equations $\left\{f_{i}, q_{j}\right\}=0$, for all indices $i, j$, may be replaced by the single equation

$$
(F-F(m)) \circ \varphi=g \circ\left(q_{1}, \ldots, q_{n}\right),
$$


where $\varphi=\left(x_{1}, \ldots, x_{n}, \xi_{1}, \ldots, \xi_{n}\right)^{-1}$ and $g$ is a diffeomorphism from a small neighborhood of the origin in $\mathbb{R}^{n}$ into another such neighborhood, such that $g(0, \ldots, 0)=$ $(0, \ldots, 0)$.

If the dimension of $M$ is 4 and $F$ has no hyperbolic singularities - which is the case which is most important to us in this paper - we have the following possibilities for the map $\left(q_{1}, q_{2}\right)$, depending on the rank of the critical point:

(1) If $m$ is a critical point of $F$ of rank zero, then $q_{j}$ is one of

(i) $q_{1}=\left(x_{1}^{2}+\xi_{1}^{2}\right) / 2$ and $q_{2}=\left(x_{2}^{2}+\xi_{2}^{2}\right) / 2$.

(ii) $q_{1}=x_{1} \xi_{2}-x_{2} \xi_{1}$ and $q_{2}=x_{1} \xi_{1}+x_{2} \xi_{2}$.

On the other hand,

(2) If $m$ is a critical point of $F$ of rank one, then

(iii) $q_{1}=\left(x_{1}^{2}+\xi_{1}^{2}\right) / 2$ and $q_{2}=\xi_{2}$.

In this case, a nondegenerate critical point is called elliptic-elliptic, transversally elliptic, or focus-focus, respectively, if both components $q_{1}, q_{2}$ are of elliptic type, one component is of elliptic type and the other component is $\xi$, or $q_{1}, q_{2}$ together correspond to a focus-focus component.

The analytic case of Eliasson's theorem was proved by Rüßmann 91 for systems with two degrees of freedom $(2 n=4)$ and by Vey 105 in any dimension. In the $\mathrm{C}^{\infty}$ category the lemme de Morse isochore of Colin de Verdière and Vey [24] implies Eliasson's result for systems with one degree of freedom. Eliasson's proof of the general case was somewhat loose at a crucial step, but recently this has been clarified [78, 112].

4.2.2. Degenerate critical points. Degenerate critical points appear in many applications, i.e., rigid body dynamics. The study of degenerate critical points of integrable systems is difficult, and little is known in general. A few particular situations are relatively understood. For analytic systems with one degree of freedom a more concrete method is presented in 25]. A general linearization result in the analytic category is given in [118. Another result may be found in [116. Further studies of degenerate singularities may be found in Kalashnikov [57, where a semiglobal topological classification of stable degenerate singularities of corank 1 for systems with two degrees of freedom is given; in Bolsinov, Fomenko, and Richter [13], where it was shown how semiglobal topological invariants of degenerate singularities can be used to describe global topological invariants of integrable systems with two degrees of freedom, and in Nekhoroshev, Sadovskii, and Zhilinskii 80, where the so-called fractional monodromy phenomenon is explained via topological properties of degenerate singularities corresponding to higher-order resonances. The best context in which to approach these singularities is probably algebraic geometry, and we hope that this article may bring some additional interactions between algebraic geometers and specialists on integrable systems. Interactions have begun to develop in the context of mirror symmetry, where the study of singular Lagrangian fibrations is relevant [54, 55, 53, 52, 15, 16, 17. From the viewpoint of algebraic singularity theory, several interesting results concerning the deformation complex of Lagrangian varieties have been discovered recently [94, 49.

Throughout this paper, and unless otherwise stated, we assume that all singularities are nondegenerate. 


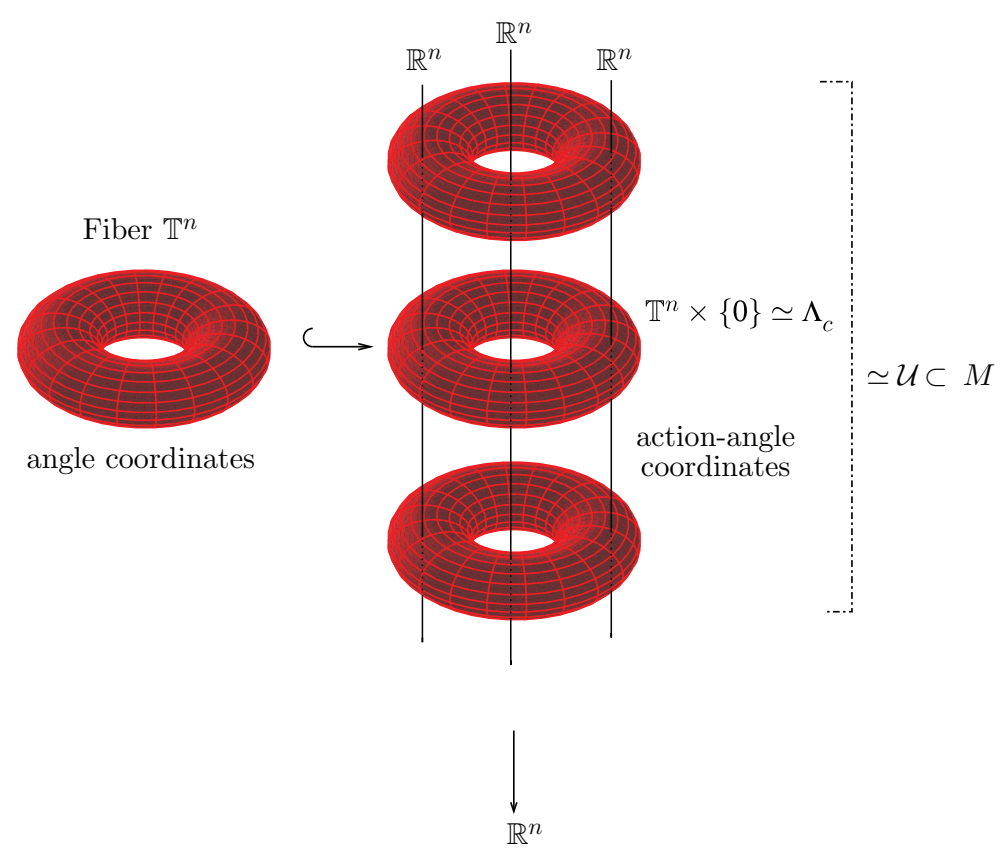

action coordinates

FiguRE 5.1. According to the Liouville-Arnold-Mineur theorem, a tubular neighborhood $\mathcal{U}$ of a regular fiber $\Lambda_{c}$ embeds symplectically into $\mathrm{T}^{*}\left(\mathbb{T}^{n}\right) \simeq \mathbb{T}^{n} \times \mathbb{R}^{n}$.

\section{Semiglobal theory of COMPletely integrable Systems}

If one aims at understanding the geometry of a completely integrable foliation or its microlocal analysis, the semiglobal aspect is probably the most fundamental. The terminology "semiglobal" refers to any term that applies to an invariant neighborhood of a leaf of the foliation. This semiglobal study is what allows, for instance, the construction of quasi-modes associated to a Lagrangian submanifold. Sometimes semiglobal merely reduces to local, when the leaf under consideration is a critical point with only elliptic blocks.

5.1. Regular fibers. The analysis of neighborhoods of regular fibers, based on the so-called Liouville-Arnold-Mineur theorem (also known as the action-angle theorem), is now routine and fully illustrated in the literature, for classical aspects as well as for quantum ones. It is the foundation of the whole modern theory of completely integrable systems in the spirit of Duistermaat's seminal article 31, but also of KAM-type perturbation theorems.

The microlocal analysis of action-angle variables starts with the work of Colin de Verdière [21, followed in the $\hbar$ semiclassical theory by Charbonnel [18, and more recently by Vũ Ngọc as well as various articles by Zelditch, Toth, Popov, Sjöstrand, and many others. The case of compact symplectic manifolds has recently been started, using the theory of Toeplitz operators 20. 
Let $F=\left(f_{1}, \ldots, f_{n}\right)$ be an integrable system on a symplectic manifold $M$. For the remainder of this article we shall assume $F$ to be proper, in which case all fibers are compact. Let $c$ be a regular value of $F$. If we restrict ourselves to an adequate invariant open set, we can always assume that the fibers of $F$ are connected. Let $\Lambda_{c}:=F^{-1}(c)$. The fibers of $F$ are compact orbits of a locally free $\mathbb{R}^{n}$-action given by the vector fields $\mathcal{H}_{f_{i}}$, so they are tori. In what follows, we identify $\mathrm{T}^{*} \mathbb{T}^{n}$ with $\mathbb{T}^{n} \times \mathbb{R}^{n}$, where $\mathbb{T}=\mathbb{R} / \mathbb{Z} \simeq S^{1}$, equipped with coordinates $\left(x_{1}, \ldots, x_{n}, \xi_{1}, \ldots, \xi_{n}\right)$ such that the canonical Liouville 1-form is $\sum_{i} \xi_{i} \mathrm{~d} x_{i}$.

Theorem 5.1 (Liouville-Arnold-Mineur 75, 4). If $\Lambda_{c}$ is regular, there exists a local symplectomorphism $\chi$ from the cotangent bundle $\mathrm{T}^{*} \mathbb{T}^{n}$ of $\mathbb{T}^{n}$ into $M$ sending the zero section onto the regular fiber $\Lambda_{c}$ in such a way that that

$$
F \circ \chi=\varphi \circ\left(\xi_{1}, \ldots, \xi_{n}\right)
$$

for $\varphi$ a local diffeomorphism of $\mathbb{R}^{n}$.

In this statement, $\chi$ is a symplectomorphism defined on a neighborhood of the zero section $\{\xi=0\}$, with values in a saturated neighborhood of the torus $\Lambda_{c}$. On the other hand, $\varphi$ is a diffeomorphism defined in a neighborhood of the origin in $\mathbb{R}^{n}$, and $\varphi(0)=F\left(\Lambda_{c}\right)$.

It is important to remark that $\mathrm{d} \varphi$ is an invariant of the system since it is determined by periods of periodic trajectories of the initial system. Regarded as functions on $M$ the $\xi_{j}$ 's are called action variables of the system for one can find a primitive $\alpha$ of $\omega$ in a neighborhood of $\Lambda_{c}$ such that the $\xi_{j}$ 's are integrals of $\alpha$ on a basis of cycles of $\Lambda_{c}$ depending smoothly on $c$. The coordinates $\left(x_{1}, \ldots, x_{n}, \xi_{1}, \ldots, \xi_{n}\right)$ are known as action-angle variables. See Figure 5.1 for an illustration of Theorem 5.1. See [68] for a version of the action-angle theorem in the case of Poisson manifolds.

5.2. Singular fibers. This section is devoted to the semiglobal structure of fibers with nondegenerate singularities. We are only aware of a very small number of semiglobal results for degenerate singularities, as mentioned in Section 4.2 .2 .

The topological analysis of nondegenerate singular fibers was mainly initiated by Fomenko [46] and was successfully expanded by a number of his students; cf. [12]. As far as we know, Lerman and Umanskii [69, 70] were certainly among the first authors to systematically study critical points of Poisson actions on symplectic 4manifolds; their paper [69 is an English translation of their original paper which was published in a Russian journal. This article is probably the first where focusfocus singularities are treated in detail. These works by Lerman and Umanskii have had an important influence on the Fomenko school. We would also like to mention that M. Kharlamov appears to be the first author to systematically do a topological analysis of integrable systems in rigid-body dynamics. His results and methods 60 were precursors of various aspects of the mathematical theory of Hamiltonian systems that we discuss in the present paper (unfortunately 60 has not been translated into English yet, but some of the references therein refer to English papers of Kharmalov where his original results may be found).

5.2.1. Elliptic case. Near an elliptic fixed point, the fibers are small tori and are entirely described by the local normal form, for classical systems as well as for semiclassical ones (the system is reduced to a set of uncoupled harmonic oscillators). Therefore we shall not talk about this type of singularity any further, even if, strictly speaking, the semiglobal semiclassical study has not been fully carried out 
for transversally elliptic singularities. But no particular difficulties are expected in that case.

5.2.2. Hyperbolic case. Just as elliptic blocks, hyperbolic blocks have one degree of freedom (normal form $q_{i}=x_{i} \xi_{i}$ ), but they turn out to be more complicated. However, in the particular case where $M$ is a surface, there is a classification due to Dufour, Molino, and Toulet, which we present next. In addition to their result, this classification is interesting to us because it introduces a way to construct symplectic invariants which is similar to the way symplectic invariants are constructed for focus-focus singularities. (This will be key to the study of semitoric integrable systems in Sections 6, 18, of this paper). Moreover, using this classification as a stepping stone, Dufour, Molino, and Toulet gave a global symplectic classification of completely integrable systems on surfaces, which serves as an introduction to the recent classification of semitoric integrable systems on symplectic 4-manifolds given later in the paper.

Two completely integrable systems with one degree of freedom $\left(M, \omega, f_{1}\right)$ and $\left(M, \omega^{\prime}, f_{1}^{\prime}\right)$ are isomorphic if there exists a symplectomorphism $\chi: M \rightarrow M^{\prime}$ and a smooth map $g$ such that $\chi^{*} f_{1}^{\prime}=g \circ f_{1}$. A nondegenerate critical point $p$ of $\left(M, \omega, f_{1}\right)$ is either elliptic or hyperbolic (there cannot be focus-focus points).

If $p$ is elliptic, there exist local coordinates $(x, y)$ and a function $g$ in a neighborhood $V$ of $p$ such that $f_{1}=g\left(x^{2}+y^{2}\right)$ and $\omega=\mathrm{d} x \wedge \mathrm{d} y$, so geometrically the integral curves of the Hamiltonian vector field $\mathcal{H}_{f_{1}}$ generated by $f_{1}$ are concentric circles centered at $p$. If $p$ is hyperbolic, there exist local coordinates $(x, y)$ in a neighborhood $U$ of $p$ and a function $h$ such that $f_{1}=h(x y)$ and $\omega=\mathrm{d} x \wedge \mathrm{d} y$. In this case the integral curves of $\mathcal{H}_{f_{1}}$ are hyperboloid branches $x y=$ constant. One usually calls these integral curves the leaves of the foliation induced by $f_{1}$. We

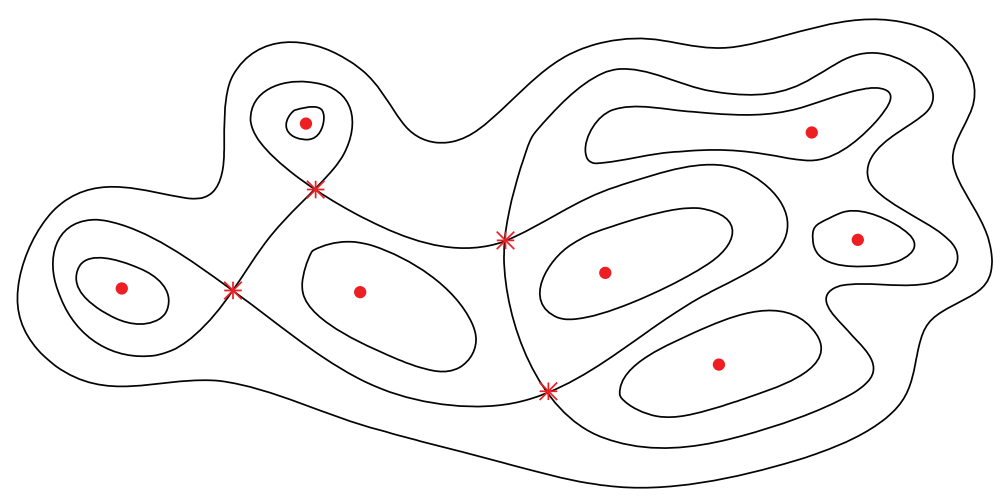

Figure 5.2. Elliptic and hyperbolic singularities on a surface. The figure shows how in the same leaf there may be several singularities, though we make the generic assumption that there is at most one singularity per fiber. Hyperbolic singularities are represented by a red star, elliptic singularities are represented by a red dot. Note how nearby a hyperbolic singularity the local model looks, as in Figure 5.3. Around an elliptic singularity, the leaves are concentric circles around the singularity. 


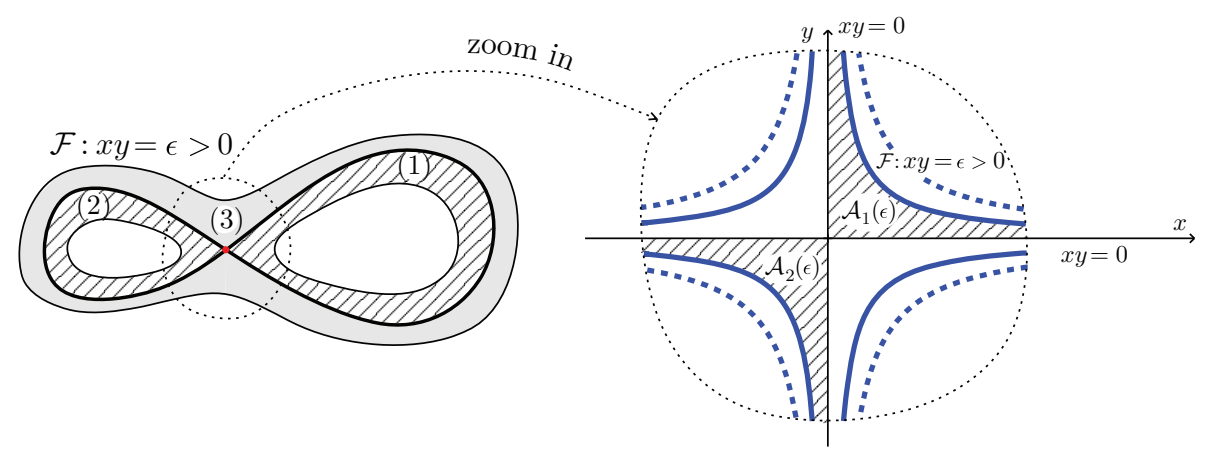

Figure 5.3. An enlargement of a hyperbolic singularity at the intersection of the $x$ and $y$ axes.

make the generic assumption that our systems may have at most one singularity per leaf of the induced foliation.

The saturation of $U$ by the foliation has the appearance of an enlarged figureeight with three components: (1) and (2) corresponding to $x y>0$ and (3) corresponding to $x y<0$. See Figures 5.3 and 5.2 , where therein $\mathcal{F}$ denotes the entire leaf of the foliation generated by $f_{1}$ defined in local coordinates near $p$ by $x y=\epsilon>0$, for some $\epsilon>0$. The area in region (1) between $\mathcal{F}$ and the figure-eight defined locally by $x y=0$ is given by $\mathcal{A}_{1}(\epsilon)=-\epsilon \ln (\epsilon)+h_{1}(\epsilon)$ for some smooth function $h_{1}=h_{1}(\epsilon)$. Similarly, the area in region $(2)$ between $\mathcal{F}$ and the figure-eight $x y=0$ is given by $\mathcal{A}_{2}(\epsilon)=-\epsilon \ln (\epsilon)+h_{2}(\epsilon)$ for some smooth function $h_{2}=h_{2}(\epsilon)$, and the area in region $(3)$ between $\mathcal{F}$ and the figure-eight is given by $\mathcal{A}_{3}(\epsilon)=2 \epsilon \ln |\epsilon|+h(\epsilon)$ for some smooth function $h=h(\epsilon)$. In addition, for each $q$,

$$
h^{(q)}=-h_{1}^{(q)}(0)+h_{2}^{(q)}(0) .
$$

The Taylor series at 0 of $h_{1}$ and $h_{2}$ are symplectic invariants of $\left(M, \omega, f_{1}\right)$; cf. 38, Proposition 1].

Let $\mathcal{G}$ be the topological quotient of $M$ by the relation $a \sim b$ if and only if $a$ and $b$ are in the same leaf of the foliation induced by $f_{1}$. The space $\mathcal{G}$ is called the Reeb graph of $M$. Let $\pi: M \rightarrow \mathcal{G}$ be the canonical projection map. In this context we call a regular point the image by $\pi$ of a regular leaf, a bout the image of an elliptic point, and a bifurcation point the image of a figure-eight leaf. The edges are the parts of $\mathcal{G}$ contained between two singular points. If $s$ is a bifurcation point, then it has three edges, one of which corresponds to the leaves extending along the separatrix. We say that this edge is the trunk of $s$. The two other edges are the branches of $s$. The graph $\mathcal{G}$ is provided with the measure $\mu$, the image by $\pi$ of the measure defined by the symplectic form $\omega$ on $M$.

Let $p \in M$ be a hyperbolic point, and let $(x, y)$ be the aforementioned local coordinates in a neighborhood of $p$. We define a function $\epsilon=x y$ in a neighborhood of the corresponding bifurcation point $s$, such that $x y>0$ on the branches of $s$ and 
$x y<0$ on the trunk of $s$. The expression of $\mu$ in this neighborhood is

$$
\left\{\begin{aligned}
\mathrm{d} \mu_{i}(\epsilon)= & \left(\ln (\epsilon)+g_{i}(\epsilon)\right) \mathrm{d} \epsilon \text { on each branch } i=1,2, \\
\mathrm{~d} \mu(\epsilon)= & (2 \ln |\epsilon|+g(\epsilon)) \mathrm{d} \epsilon \text { on the trunk, with } g, g_{1}, g_{2} \\
& \text { smooth functions satisfying, for each } q, \\
g^{(q)}(0)= & \left(\left(g_{1}^{(q)}(0)+g_{2}^{(q)}(0)\right) .\right.
\end{aligned}\right.
$$

It follows from [38, Proposition 1] stated above that the Taylor series at 0 of $g_{1}, g_{2}$ are invariants of $(\mathcal{G}, \mu)$.

Definition 5.2 (Définition 1 in 38). Let $\mathcal{G}$ be a topological 1-complex whose vertices have degrees 1 or 3 . For each degree 3 vertex $s$, which one calls a bifurcation point, one distinguishes an edge and calls it the trunk of $s$; the two others are the branches of $s$. We provide $\mathcal{G}$ with an atlas of the following type.

- Outside of the bifurcation points, it is a classical atlas of a manifold with boundary of dimension 1.

- In a neighborhood of each bifurcation point $s$, there exists an open set $V$ and a continuous map $\varphi: V \rightarrow(-\epsilon, \epsilon), \epsilon>0$, with $\varphi(s)=0$ and such that, if $T$ is the trunk of $s$ and $B_{1}, B_{2}$ are the branches of $s,\left.\varphi\right|_{B_{i}}$ is bijective on $[0, \epsilon), i=1,2$ and $\left.\varphi\right|_{T}$ is bijective on $(-\epsilon, 0]$. We require that the changes of charts are smooth on each part $T \cup B_{i}, i=1,2$.

The topological 1-complex $\mathcal{G}$ is provided with a measure given by a nonzero density, smooth on each edge, and such that for each vertex $s$ of degree 3 , there exists a chart $\varphi$ at $s$ in which the measure $\mu$ is written as in equation (5.1). We denote by $(\mathcal{G}, \mathcal{D}, \mu)$ such a graph provided with its smooth structure and its measure, and we call it an affine Reeb graph. An isomorphism of such a graph is a bijection preserving the corresponding smooth structure and measure.

One can show [38, Lemme 2] that if the measure $\mu$ is written in another chart $\tilde{\varphi}$ in a neighborhood of $s$,

$$
\begin{aligned}
\mathrm{d} \mu_{i}(\epsilon) & =\left(\ln (\epsilon)+\tilde{g}_{i}(\epsilon)\right) \mathrm{d} \epsilon \quad \text { on each branch } B_{i} \text { of } s, \\
\mathrm{~d} \mu(\epsilon) & =(2 \ln |\epsilon|+\tilde{g}(\epsilon)) \mathrm{d} \epsilon \quad \text { on the trunk of } s,
\end{aligned}
$$

then the functions $g_{i}$ and $\tilde{g}_{i}$ have the same Taylor series at the origin (hence the Taylor series of $g$ and $\tilde{g}$ are equal), which shows that the Taylor series of the functions $g_{i}$ give invariants for the bifurcation points.

Let $\mathcal{G}$ be a combinatorial graph with vertices of degree 1 and of degree 3. For each vertex $s$ of degree 3 , one distinguishes in the same fashion as in Definition 5.2 the trunk and the branches of $s$, and one associates to each a sequence of real numbers. In addition, to each edge one associates a positive real number, called its length. Such a graph is called a weighted Reeb graph. To each affine Reeb graph one naturally associates a weighted Reeb graph, the sequence of numbers associating to the branches corresponding to coefficients of the Taylor series of the functions $g_{i}$. The lengths of the edges are given by their measure.

These considerations tell us the first part of the the following beautiful classification theorem.

Theorem 5.3 (Dufour, Molino, and Toulet [38]). One can associate to a triplet $\left(M, \omega, f_{1}\right)$ an affine Reeb graph $(\mathcal{G}, \mathcal{D}, \mu)$, which is unique up to isomorphisms, and to such an affine Reeb graph a weighted Reeb graph, unique up to isomorphisms. Conversely, every weighted Reeb graph is the graph associated to an affine Reeb 
graph, unique up to isomorphisms, and every affine Reeb graph is the Reeb graph associated to a triplet $\left(M, \omega, f_{1}\right)$, unique up to isomorphisms.

The higher-dimensional case will not be treated in general in the present paper, as we will assume our systems do not have hyperbolic singularities.

5.2.3. Focus-focus case. Unless otherwise stated, for the remainder of the paper we focus on the case where the symplectic manifold $M$ is 4 dimensional. Eliasson's theorem gives the local structure of focus-focus singularities. Several people noticed in the years 1992-1997 that this was enough to determine the monodromy of the foliation around the singular fiber. Actually, this local structure is a starting point for understanding much more: the semiglobal classification of a singular fiber of focus-focus type. Unlike monodromy which is a topological invariant already observed in torus fibrations without Hamiltonian structure, the semiglobal classification involves purely symplectic invariants. We proceed to describe this semiglobal classification, which complements Eliasson's theorem, in two steps.

(a) Application of Eliasson's theorem. Let $F=\left(f_{1}, f_{2}\right)$ be a completely integrable system with two degrees of freedom on a four-dimensional symplectic manifold $M$. Let $\mathcal{F}$ be the associated singular foliation to the completely integrable system $F=\left(f_{1}, f_{2}\right)$, the leaves of which are by definition the connected components of the fibers $F^{-1}(c)$ of $F: M \rightarrow \mathbb{R}^{2}$. Let $m$ be a critical point of focus-focus type. We assume for simplicity that $F(m)=0$, and that the (compact, connected) fiber $\Lambda_{0}:=F^{-1}(0)$ does not contain other critical points. One can show that $\Lambda_{0}$ is a "pinched" toru 8 surrounded by regular fibers which are standard 2-tori; see Figure 5.4. What are the semiglobal invariants associated to this singular fibration?

One of the major characteristics of focus-focus singularities is the existence of a Hamiltonian action of $S^{1}$ that commutes with the flow of the system, in a neighborhood of the singular fiber that contains $m$. By Eliasson's theorem [43, 112, there exist symplectic coordinates $(x, y, \xi, \eta)$ in a neighborhood $U$ around $m$ in which $\left(q_{1}, q_{2}\right)$, given by

$$
q_{1}=x \eta-y \xi, q_{2}=x \xi+y \eta,
$$

is a momentum map for the foliation $\mathcal{F}$; here the critical point $m$ corresponds to coordinates $(0,0,0,0)$. Fix $A^{\prime} \in \Lambda_{0} \cap(U \backslash\{m\})$, and let $\Sigma$ denote a small two-dimensional surface transversal to $\mathcal{F}$ at the point $A^{\prime}$.

Since the Liouville foliation in a small neighborhood of $\Sigma$ is regular for both $F$ and $q=\left(q_{1}, q_{2}\right)$, there is a diffeomorphism $\varphi$ from a neighborhood $U^{\prime}$ of $F\left(A^{\prime}\right) \in \mathbb{R}^{2}$ into a neighborhood of the origin in $\mathbb{R}^{2}$ such that $q=\varphi \circ F$. Thus there exists a smooth momentum map $\Phi=\varphi \circ F$ for the foliation, defined on a neighborhood $\Omega=F^{-1}\left(U^{\prime}\right)$ of $\Lambda_{0}$, which agrees with $q$ on $U$.

Write $\Phi:=\left(H_{1}, H_{2}\right)$ and $\Lambda_{z}:=\Phi^{-1}(c)$. Note that $\Lambda_{0}=\mathcal{F}_{m}$. It follows from (5.2) that near $m$ the $H_{1}$-orbits must be periodic of primitive period $2 \pi$, whereas the vector field $\mathcal{H}_{H_{2}}$ is hyperbolic with a local stable manifold (the $(\xi, \eta)$-plane) transversal to its local unstable manifold (the $(x, y)$-plane). Moreover, $\mathcal{H}_{H_{2}}$ is radial, meaning that the flows tending towards the origin do not spiral on the local (un)stable manifolds.

(b) Symplectic semiglobal classification of focus-focus point. Suppose that $A \in \Lambda_{c}$ for some regular value $c$. Let $\tau_{2}(c)>0$ be the time it takes the Hamiltonian flow

\footnotetext{
${ }^{8}$ Lagrangian immersion of a sphere $S^{2}$ with a transversal double point.
} 


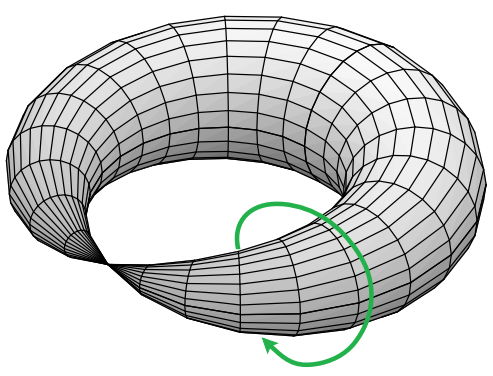

FIGURE 5.4. Focus-focus singularity and vanishing cycle for the pinched torus.

associated with $H_{2}$ leaving from $A$ to meet the Hamiltonian flow associated with $H_{1}$ which passes through $A$. The existence of $\tau_{2}$ is ensured by the fact that the flow of $\mathrm{H}_{2}$ is a quasi-periodic motion always transversal to the $S^{1}$-orbits generated by $H_{1}$.

Let $\tau_{1}(c) \in \mathbb{R} / 2 \pi \mathbb{Z}$ be the time it takes to go from this intersection point back to $A$, closing the trajectory.

The commutativity of the flows ensures that $\tau_{1}(c)$ and $\tau_{2}(c)$ do not depend on the initial point $A$. Indeed, if $\varphi_{1}^{t}, \varphi_{2}^{t}$ denote the Hamiltonian flows of $H_{1}$ and $H_{2}$, respectively, then we have $A=\varphi_{1}^{\tau_{1}(c)} \circ \varphi_{2}^{\tau_{2}(c)}(A)$. If $\tilde{A} \in \Lambda_{c}$ is close to $A$, the fact that $\mathrm{d} H_{1}$ and $\mathrm{d} H_{2}$ are independent near $A$ implies that the corresponding Hamiltonian action is locally free on $\Lambda_{c}$ : there exist small times $\left(t_{1}, t_{2}\right) \in \mathbb{R}^{2}$ such that

$$
\tilde{A}=\varphi_{1}^{t_{1}} \circ \varphi_{2}^{t_{2}}(A) .
$$

Thus we may write

$$
\tilde{A}=\varphi_{1}^{t_{1}+\tau_{1}(c)} \circ \varphi_{2}^{t_{2}+\tau_{2}(c)}(A)=\varphi_{1}^{\tau_{1}(c)} \circ \varphi_{2}^{\tau_{2}(c)}(\tilde{A}) .
$$

This shows that the times $\tau_{1}, \tau_{2}$ that would be obtained starting from $\tilde{A}$ are the same as those we obtained starting from $A$.

Write $c=\left(c_{1}, c_{2}\right)=c_{1}+\mathrm{i} c_{2}\left(c_{1}, c_{2} \in \mathbb{R}\right)$, and let $\ln c$ be a fixed determination of the logarithmic function on the complex plane. Let

$$
\left\{\begin{array}{l}
\sigma_{1}(c)=\tau_{1}(c)-\Im(\ln c) \\
\sigma_{2}(c)=\tau_{2}(c)+\Re(\ln c),
\end{array}\right.
$$

where $\Re$ and $\Im$ stand for the real and imaginary parts, respectively, of a complex number. Vũ Ngọc proved in [107, Proposition 3.1] that $\sigma_{1}$ and $\sigma_{2}$ extend to smooth and single-valued functions in a neighborhood of 0 and that the differential 1-form $\sigma:=\sigma_{1} \mathrm{~d} c_{1}+\sigma_{2} \mathrm{~d} c_{2}$ is closed. Notice that it follows from the smoothness of $\sigma_{1}$ that one may choose the lift of $\tau_{1}$ to $\mathbb{R}$ such that $\sigma_{1}(0) \in[0,2 \pi)$. This is the convention used throughout.

Following [107, Def. 3.1], let $S$ be the unique smooth function defined around $0 \in \mathbb{R}^{2}$ such that

$$
\mathrm{d} S=\sigma, \quad S(0)=0 .
$$

The Taylor expansion of $S$ at $(0,0)$ is denoted by $(S)^{\infty}$.

Loosely speaking, one of the components of the system is indeed $2 \pi$-periodic, but the other one generates an arbitrary flow which turns indefinitely around the 
focus-focus singularity, deviating from periodic behavior in a logarithmic fashion, up to a certain error term; this deviation from being logarithmic is the symplectic invariant $(S)^{\infty}$.

Theorem 5.4 (Vũ Ngọc [107]). The Taylor series expansion $(S)^{\infty}$ is well defined (it does not depend on the choice of Eliasson's local chart). Moreover, it classifies the singular foliation in a neighborhood of $\Lambda_{0}$. This means that another system has the same Taylor series invariant near a focus-focus singularity if and only if there is a symplectomorphism which takes a foliated neighborhood of the singular fiber to a foliated neighborhood of the singular fiber preserving the leaves of the foliation and sending the singular fiber to the singular fiber.

Conversely, if $\Sigma$ is any formal series in $\mathbb{R} \llbracket X, Y \rrbracket$ with $X$-coefficient in $[0,2 \pi)$ and without constant term, then there exists a singular foliation of focus-focus type whose Taylor series expansion is $\Sigma$.

The fact that two focus-focus fibrations are always semiglobally topologically conjugate was already proved by Lerman and Umanskii [69] and Nguyên Tiên Zung [115, who introduced various topological notions of equivalence.

$S$ can be interpreted as a regularized (or desingularized) action. Indeed if $\gamma_{z}$ is the loop on $\Lambda_{z}$ defined just as in the description of $\tau_{j}$ above, and if $\alpha$ is a semiglobal primitive of the symplectic form $\omega$, let $\mathcal{A}(z)=\int_{\gamma_{c}} \alpha$. Then

$$
S(z)=\mathcal{A}(z)-\mathcal{A}(0)+\mathfrak{R}(z \ln z-z) .
$$

5.3. Example. One can check that the singularities of the coupled spin-oscillator $S^{2} \times \mathbb{R}^{2}$ model mentioned in Section 1 (equipped with the product symplectic form or the standard area forms) are nondegenerate and of elliptic-elliptic, transversally elliptic, or focus-focus type.

It has exactly one focus-focus singularity at the "North Pole" $(((0,0,1),(0,0)) \in$ $\left.S^{2} \times \mathbb{R}^{2}\right)$ and one elliptic-elliptic singularity at the "South Pole" $((0,0,-1),(0,0))$. Let us parametrize the singular fiber $\Lambda_{0}:=F^{-1}(1,0)$. This singular fiber $\Lambda_{0}$ corresponds to the system of equations $J=1$ and $H=0$, which is given explicitly by a system of two nonlinear equations $J=\left(u^{2}+v^{2}\right) / 2+z=0$ and $H=\frac{1}{2}(u x+v y)=0$ on the coordinates $(x, y, z, u, v)$.

In order to solve this system of equations one introduces polar coordinates $u+\mathrm{i} v=r \mathrm{e}^{\mathrm{i} t}$ and $x+\mathrm{i} y=\rho \mathrm{e}^{\mathrm{i} \theta}$, where we recall that the 2 -sphere $S^{2} \subset \mathbb{R}^{3}$ is equipped with coordinates $(x, y, z)$ and $\mathbb{R}^{2}$ is equipped with coordinates $(u, v)$. For $\epsilon= \pm 1$, we consider the mapping

$$
S_{\epsilon}:[-1,1] \times \mathbb{R} / 2 \pi \mathbb{Z} \rightarrow \mathbb{R}^{2} \times S^{2}
$$

given by the formula

$$
S_{\epsilon}(p)=\left(r(p) \mathrm{e}^{\mathrm{i} t(p)},\left(\rho(p) \mathrm{e}^{\mathrm{i} \theta(p), z(p)}\right)\right),
$$

where $p=(\tilde{z}, \tilde{\theta}) \in[-1,1] \times[0,2 \pi)$ and

$$
\left\{\begin{array}{l}
r(p)=\sqrt{2(1-\tilde{z})}, \\
t(p)=\tilde{\theta}+\epsilon \frac{\pi}{2}, \\
\rho(p)=\sqrt{1-\tilde{z}^{2}} \\
\theta(p)=\tilde{\theta} \\
z(p)=\tilde{z} .
\end{array}\right.
$$


Then the map $S_{\epsilon}$, where $\epsilon= \pm 1$, is continuous, and $S_{\epsilon}$ restricted to $(-1,1) \times$ $\mathbb{R} / 2 \pi \mathbb{Z}$ is a diffeomorphism onto its image. If we let $\Lambda_{0}^{\epsilon}:=S_{\epsilon}([-1,1] \times \mathbb{R} / 2 \pi \mathbb{Z})$, then $\Lambda_{0}^{1} \cup \Lambda_{0}^{2}=\Lambda_{0}$ and

$$
\Lambda_{0}^{1} \cap \Lambda_{0}^{2}=(\{(0,0)\} \times\{(1,0,0)\}) \cup\left(C_{2} \times\{(0,0,-1)\}\right),
$$

where $C_{2}$ denotes the circle of radius 2 centered at $(0,0)$ in $\mathbb{R}^{2}$. Moreover, $S_{\epsilon}$ restricted to $(-1,1) \times \mathbb{R} / 2 \pi \mathbb{Z}$ is a smooth Lagrangian embedding into $\mathbb{R}^{2} \times S^{2}$. The singular fiber $\Lambda_{0}$ consists of two sheets glued along a point and a circle; topologically $\Lambda_{0}$ is a pinched torus, i.e., a two-dimensional torus $S^{1} \times S^{1}$ in which one circle $\{p\} \times S^{1}$ is contracted to a point (which is of course not a a smooth manifold at the point which comes from the contracting circle). These statements correspond to [89, Proposition 2.8].

It was proven in [89. Theorem 1.1] that the linear deviation from exhibiting logarithmic behavior in a saturated neighborhood of the focus-focus singularity is given by the linear map $L: \mathbb{R}^{2} \rightarrow \mathbb{R}$ with expression

$$
L(X, Y)=5 \ln 2 X+\frac{\pi}{2} Y .
$$

In other words, we have an equality

$$
(S(X, Y))^{\infty}=L(X, Y)+\mathcal{O}\left((X, Y)^{2}\right) .
$$

This computation is involved and uses some deep formulas from microlocal analysis proved in the late 1990s. At the time of writing this paper, we do not have a strategy to compute the higher-order terms of the Taylor series invariant.

5.4. Applications. Theorem 5.4leads to a number of applications, which although they are outside the scope of this paper, we briefly note. One can, for instance, exploit the fact that the set of symplectic equivalence classes of these foliations acquires a vector space structure. That is what Symington does in 99 to show that neighborhoods of focus-focus fibers are always symplectomorphic (after forgetting the foliation, of course). For this one introduces functions $S_{0}$ and $S_{1}$ whose Taylor expansions give the invariants of the two foliations, and which constructs a "path of foliations" by interpolating between $S_{0}$ and $S_{1}$. Then a Moser-type argument yields the result (since the symplectic forms are cohomologous).

The theorem is also useful for doing calculations in a neighborhood of the fiber. For instance, in this way it is possible to determine the validity of nondegeneracy conditions that appear in KAM-type theorems 9 for a perturbation of a completely integrable system with a focus-focus singularity (see also [114]).

Theorem 5.5 (Dullin and Vũ Ngọc 39]). Let H be a completely integrable Hamiltonian with a loxodromic singularity at the origin (i.e., $H$ admits a singular Lagrangian foliation of focus-focus type at the origin). Then Kolmogorov's nondegeneracy condition is fulfilled on all tori close to the critical fiber, and the "isoenergetic turning frequencies" condition is fulfilled except on a one-parameter family of tori corresponding to a curve through the origin in the image of the momentum map which is transversal to the lines of constant energy $H$.

\footnotetext{
${ }^{9} \mathrm{~A}$ nice discussion of these various conditions can be found in 90 .
} 
5.5. A topological classification. The present paper is devoted to the symplectic theory of Hamiltonian integrable systems. A large number authors have made contributions to the topological theory of Hamiltonian integrable systems, in particular Fomenko and his students, and Nguyên Tiên Zung. In this section we briefly present a classification result due to Zung, which holds in any dimension and for all so-called topologically stable (nondegenerate) singularities. For precise statements we refer to Zung [115, Section 7].

Let $\mathcal{F}$ be a singular leaf (fiber corresponding to a nondegenerate singularity) of an integrable system. In what follows, a tubular neighborhood $\mathcal{U}(\mathcal{F})$ of $\mathcal{F}$ means an appropriately chosen sufficiently small saturated tubular neighborhood. We denote by $(\mathcal{U}(\mathcal{F}), \mathcal{L})$ the Lagrangian foliation in a tubular neighborhood $\mathcal{U}(\mathcal{F})$ of $\mathcal{F}$. The leaf $\mathcal{F}$ is a deformation retract of $\mathcal{U}(\mathcal{F})$.

Let $\mathcal{F}_{1}, \mathcal{F}_{2}$ be (nondegenerate) singular leaves of two integrable systems, of coranks $k_{1}, k_{2}$, respectively, and let $\left(\mathcal{U}\left(\mathcal{F}_{1}\right), \mathcal{L}_{1}\right)$ and $\left(\mathcal{U}\left(\mathcal{F}_{2}\right), \mathcal{L}_{2}\right)$ be the corresponding Lagrangian foliations. Here a singular leaf is said to be of corank $k$ if $k$ is the maximal corank of the differential of the momentum map at critical points of the singular leaf 10 For instance a focus-focus singularity in a symplectic 4manifold has corank 2 . The direct product of these singularities is the singular leaf $\mathcal{F}=\mathcal{F}_{1} \times \mathcal{F}_{2}$ of corank $k_{1}+k_{2}$, with the associated Lagrangian foliation

$$
(\mathcal{U}(\mathcal{F}), \mathcal{L}):=\left(\mathcal{U}\left(\mathcal{F}_{1}\right), \mathcal{L}_{1}\right) \times\left(\mathcal{U}\left(\mathcal{F}_{2}\right), \mathcal{L}_{2}\right) .
$$

A (nondegenerate) singularity (or singular pair) $(\mathcal{U}(\mathcal{F}), \mathcal{L})$ of corank $k$ and Williamson type $\left(k_{\mathrm{e}}, k_{\mathrm{h}}, k_{\mathrm{f}}\right)$ of an integrable system with $n$ degrees of freedom is called of direct product type topologically if it is homeomorphic, together with the Lagrangian foliation, to the direct product

$$
\begin{aligned}
&\left(\mathcal{U}\left(\mathbb{T}^{n-k}\right), \mathcal{L}_{\mathrm{r}}\right) \times(\left.\mathrm{P}^{2}\left(\mathcal{F}_{1}\right), \mathcal{L}_{1}\right) \times \cdots \times\left(\mathrm{P}^{2}\left(\mathcal{F}_{k_{\mathrm{e}}+k_{\mathrm{h}}}\right), \mathcal{L}_{k_{\mathrm{e}}+k_{\mathrm{h}}}\right) \\
& \times\left(\mathrm{P}^{4}\left(\mathcal{F}_{1}^{\prime}\right), \mathcal{L}_{1}^{\prime}\right) \times \cdots \times\left(\mathrm{P}^{4}\left(\mathcal{F}_{k_{\mathrm{f}}}^{\prime}\right), \mathcal{L}_{k_{\mathrm{f}}}^{\prime}\right)
\end{aligned}
$$

where

- the tuple $\left(\mathcal{U}\left(\mathbb{T}^{n-k}, \mathcal{L}_{\mathrm{r}}\right)\right.$ denotes the Lagrangian foliation in a tubular neighborhood of a regular $(n-k)$-dimensional torus of an integrable system with $n-k$ degrees of freedom;

- the tuple $\left(\mathrm{P}^{2}\left(\mathcal{F}_{i}\right), \mathcal{L}_{i}\right), 1 \leq i \leq k_{\mathrm{e}}+k_{\mathrm{h}}$, denotes a codimension 1 (nondegenerate) surface singularity (i.e., a singularity of an integrable system with one degree of freedom);

- the tuple $\left(\mathrm{P}^{4}\left(\mathcal{F}_{i}\right), \mathcal{L}_{i}^{\prime}\right), 1 \leq i \leq k_{\mathrm{f}}$, denotes a focus-focus singularity of an integrable system with two degrees of freedom.

In this case we have $k=k_{\mathrm{e}}+k_{\mathrm{h}}+2 k_{\mathrm{f}}$. A (nondegenerate) singularity of an integrable system is of almost direct product type topologically if a finite covering of it is homeomorphic, together with the Lagrangian foliation, to a direct product singularity. Nguyên Tiên Zung proved [115, Theorem 7.3] the following classification result.

Theorem 5.6 (Nguyên Tiên Zung [115]). If $(\mathcal{U}(\mathcal{F}), \mathcal{L})$ is a (nondegenerate) topologically stable singularity of Williamson type $\left(k_{\mathrm{e}}, k_{\mathrm{h}}, k_{\mathrm{f}}\right)$ and corank $k$ of an integrable system with $n$ degrees of freedom, then it can be written homeomorphically in the form of a quotient of a direct product singularity as in expression (5.5) by

${ }^{10}$ In [115, Zung used the terminology "codimension" for the corank $k$. 
the free action of a finite group $\Gamma$ which acts component-wise on the product and acts trivially on the elliptic components.

As Nguyên Tiên Zung points out, the decomposition in Theorem[5.6 is in general not symplectic.

\section{Introduction to SEMitoric COMPLETELY INTEGRABLE SyStems}

For the remainder of this paper, we are going to focus exclusively on semitoric completely integrable systems with two degrees of freedom on 4-manifolds; for brevity we will call these simply "semitoric systems". Essentially, this means that the system is half toric, and half completely general - but nontoric singularities must be isolated; see Definition 6.1 for a precise definition. Naturally, Hamiltonian toric manifolds form a strict subclass of semitoric systems.

Semitoric systems form an important class of integrable systems, commonly found in simple physical models. Indeed, a semitoric system can be viewed as a Hamiltonian system in the presence of an $S^{1}$-symmetry [93. In our personal opinion, it is much simpler to understand the integrable system as a whole rather than writing a theory of Hamiltonian systems on Hamiltonian $S^{1}$-manifolds.

6.1. Meaning of the integrability condition. Let us recall what the general definition of an integrable system in Section 1 means in dimension 4. In this case an integrable system on $M$ is a pair of real-valued smooth functions $J$ and $H$ on $M$, for which the Poisson bracket $\{J, H\}:=\omega\left(\mathcal{H}_{J}, \mathcal{H}_{H}\right)$ identically vanishes on $M$, and the differentials $\mathrm{d} J, \mathrm{~d} H$ are almost-everywhere linearly independendent.

Of course, here $(J, H): M \rightarrow \mathbb{R}^{2}$ is the analogue of the momentum map in the case of a torus action. In some local Darboux coordinates of $M,(x, y, \xi, \eta)$, the symplectic form $\omega$ is given by $\mathrm{d} \xi \wedge \mathrm{d} x+\mathrm{d} \eta \wedge \mathrm{d} y$, and the vanishing of the Poisson brackets $\{J, H\}$ amounts to the partial differential equation

$$
\frac{\partial J}{\partial \xi} \frac{\partial H}{\partial x}-\frac{\partial J}{\partial x} \frac{\partial H}{\partial \xi}+\frac{\partial J}{\partial \eta} \frac{\partial H}{\partial y}-\frac{\partial J}{\partial y} \frac{\partial H}{\partial \eta}=0 .
$$

This condition is equivalent to $J$ being constant along the integral curves of $\mathcal{H}_{H}$ (or $H$ being constant along the integral curves of $\mathcal{H}_{J}$ ).

6.2. Singularities. We introduce the main object of the remaining part of this paper, semitoric systems, and explain what singularities can occur in these systems.

Definition 6.1. A semitoric integrable system on $M$ is an integrable system for which the component $J$ is a proper momentum map for a Hamiltonian circle action on $M$, and the associated map $F:=(J, H): M \rightarrow \mathbb{R}^{2}$ has only nondegenerate singularities in the sense of Williamson, without real-hyperbolic blocks.

Remark 6.2. There are examples which come endowed with a Hamiltonian $S^{1}$ action but do not fit Definition 6.1. The direct product $S^{2} \times S^{2}$ equipped with the Hamiltonians $J:=z_{1}$ and $H:=x_{2} y_{2}$, where $\left(x_{1}, y_{1}, z_{1}\right),\left(x_{2}, y_{2}, z_{2}\right)$ are the coordinates on the first and second copies of $S^{2}$, respectively, is a nondegenerate system with a proper $S^{1}$-momentum map $J$, but it contains hyperbolic singularities. 
Similarly, the spherical pendulum on $\mathrm{T}^{*} S^{2}$ (mentioned in Section 3.1) equipped with $J\left(\theta, \varphi, \xi_{\theta}, \xi_{\varphi}\right)=\xi_{\varphi}$ and

$$
H(\underbrace{\theta, \varphi}_{\text {sphere }}, \underbrace{\xi_{\theta}, \xi_{\varphi}}_{\text {fiber }})=\underbrace{\frac{1}{2}\left(\xi_{\theta}^{2}+\frac{1}{\sin ^{2} \theta} \xi_{\varphi}^{2}\right)}_{\text {kinetic energy }}+\underbrace{\cos \theta}_{\text {potential }}
$$

is a nondegenerate system that does not have hyperbolic singularities. However $J$ is not a proper map. We are developing a theory that deals with this situation; see [85.

Let us spell out this definition concretely. The Hamiltonian flow defined by $J$ is periodic, with fixed period $2 \pi$. The flow of $H$ is in general not periodic, but of course it is quasi-periodic on regular Liouville tori. The properness of $J$ means that the preimage by $J$ of a compact set is compact in $M$ (which is immediate if $M$ is compact). The nondegeneracy hypothesis for $F$ means that if $m$ is a critical point of $F$, then there exists an invertible 2-by-2 matrix $B$ such that, if we write $\tilde{F}=B \circ F$, one of the situations described in the following table holds in some local symplectic coordinates $(x, y, \xi, \eta)$ near $m$ in which $m=(0,0,0,0)$ and $\omega=\mathrm{d} \xi \wedge \mathrm{d} x+\mathrm{d} \eta \wedge \mathrm{d} y$.

\begin{tabular}{|l|l|}
\hline TYPE & $\widetilde{F}:=(H, J): M \rightarrow \mathbb{R}^{2}$ IN COORDINATES $(x, y, \xi, \eta)$ \\
\hline Transversally elliptic & $\widetilde{F}=\left(\eta+\mathcal{O}\left(\eta^{2}\right), \frac{1}{2}\left(x^{2}+\xi^{2}\right)+\mathcal{O}\left((x, \xi)^{3}\right)\right.$ \\
\hline Elliptic-elliptic & $\widetilde{F}=\frac{1}{2}\left(x^{2}+\xi^{2}, y^{2}+\eta^{2}\right)+\mathcal{O}\left((x, \xi, y, \eta)^{3}\right)$ \\
\hline Focus-focus & $\widetilde{F}=(x \eta-y \xi, x \xi+y \eta)+\mathcal{O}\left((x, \xi, y, \eta)^{3}\right)$ \\
\hline
\end{tabular}

In the case of semitoric systems, the Williamson types of the singularities are of the form $\left(k_{\mathrm{e}}, 0, \mathrm{k}_{\mathrm{f}}\right)$, i.e., $k_{\mathrm{h}}=0$.

Note that this is not a result in a neighborhood of a fiber.

Again, perhaps the simplest noncompact semitoric integrable system is the coupled spin-oscillator $S^{2} \times \mathbb{R}^{2}$. The component $J$ is the momentum map for the Hamiltonian circle action on $M$ which rotates simultaneously about the vertical axis of $S^{2}$ and about the origin of $\mathbb{R}^{2}$. By the Liouville-Arnold-Mineur actionangle theorem, the regular fibers are 2-tori. We saw in Section 5.3 that this system has a unique focus-focus singularity at $(0,0,1,0,0)$ with fiber a pinched torus. The other singular fibers are either circles or points. The authors have studied the symplectic and spectral theory of this system in 89 .

6.3. Convexity properties: the polygon invariant. This section analyzes to what extent the convexity theorem of Atiyah [6] and Guillemin and Sternberg 56. holds in the context of semitoric completely integrable systems. Vũ Ngọc proved in 108 that one can meaningfully associate a convex polygonal region to such a system.

6.3.1. Bifurcation diagrams. It is well established in the integrable systems community that the simplest and most natural object, which tells much about the structure of the integrable system under study, is the so-called bifurcation diagram. As a matter of fact, bifurcation diagrams may be defined in great generality as follows. Let $M$ and $N$ be smooth manifolds. Recall that a smooth map $f: M \rightarrow N$ is locally trivial at $n_{0} \in f(M)$ if there is an open neighborhood $U \subset N$ of $n_{0}$ such that 


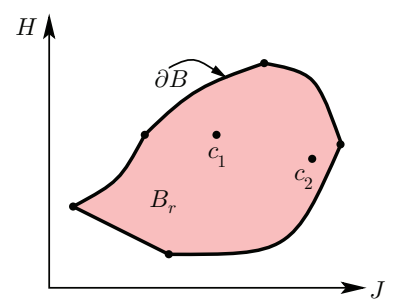

Figure 6.1. Image $F(M)$.

$f^{-1}(n)$ is a smooth submanifold of $M$ for each $n \in U$ and there is a smooth map $h: f^{-1}(U) \rightarrow f^{-1}\left(n_{0}\right)$ such that $f \times h: f^{-1}(U) \rightarrow U \times f^{-1}\left(n_{0}\right)$ is a diffeomorphism. The bifurcation set or bifurcation diagram $\Sigma_{f}$ consists of all the points of $N$ where $f$ is not locally trivial. Note, in particular, that $\left.h\right|_{f^{-1}(n)}: f^{-1}(n) \rightarrow f^{-1}\left(n_{0}\right)$ is a diffeomorphism for every $n \in U$. Also, the set of points where $f$ is locally trivial is open in $N$, so $\Sigma_{f}$ is a closed subset of $N$. It is well known that the set of critical values of $f$ is included in the bifurcation set (see [1, Proposition 4.5.1]). In general, the bifurcation set strictly includes the set of critical values. This is the case for the momentum-energy map for the two-body problem [1, §9.8]. It is well known [1, p. 340] that if $f: M \rightarrow N$ is a smooth proper map, the bifurcation set of $f$ is equal to the set of critical values of $f$.

It follows that when the map $F=(J, H): M \rightarrow \mathbb{R}^{2}$ that defines the integrable system is a proper map, the bifurcation diagram is equal to the set of critical values of $F$ inside of the image $F(M)$ of $F$. This is the case for semitoric integrable systems, since the properness of the component $J$ implies the properness of $J$. As it turns out, the arrangement of such critical values is indeed important, but other crucial invariants that are more subtle and cannot be detected from the bifurcation diagram itself are needed to understand a semitoric system $F$; we deal with these in Section 7 The authors proved [87, 88] that these invariants are enough to completely determine a semitoric system up to isomorphisms.

The proof relies on a number of remarkable results by other authors on integrable systems, including Arnold [4, Atiyah [6, Dufour and Molino [37, Eliasson [42, Duistermaat [31, Guillemin and Sternberg [56, Miranda and Zung [77, and Vũ Ngọc [107, 108. In this section we explain the so-called polygon invariant, which was originally introduced by Vũ Ngọc in [108 and can be considered an analogue (for completely integrable semitoric systems) of the convex polytope that appears in the Atiyah-Guillemin-Sternberg convexity theorem (in the context of symplectic torus actions on compact manifolds).

6.3.2. Affine structures. The plane $\mathbb{R}^{2}$ is equipped with its standard affine structure with origin at $(0,0)$ and its standard orientation. Let $\operatorname{Aff}(2, \mathbb{R}):=\operatorname{GL}(2, \mathbb{R}) \ltimes$ $\mathbb{R}^{2}$ be the group of affine transformations of $\mathbb{R}^{2}$. Let $\operatorname{Aff}(2, \mathbb{Z}):=\mathrm{GL}(2, \mathbb{Z}) \ltimes \mathbb{R}^{2}$ be the subgroup of integral-affine transformations. It was proved in 108 that a semitoric system $(M, \omega, F:=(J, H))$ has finitely many focus-focus critical values $c_{1}, \ldots, c_{m_{f}}$, that if we write $B:=F(M)$, then the set of regular values of $F$ is $\operatorname{Int}(B) \backslash\left\{c_{1}, \ldots, c_{m_{f}}\right\}$, that the boundary of $B$ consists of all images of elliptic singularities, and that the fibers of $F$ are connected. The integer $m_{f}$ was the first invariant that we associated with such a system. 
Let $\mathfrak{I}$ be the subgroup of $\operatorname{Aff}(2, \mathbb{Z})$ of those transformations which leave a vertical line invariant, or equivalently, an element of $\mathfrak{I}$ is a vertical translation composed with a matrix $T^{k}$, where $k \in \mathbb{Z}$ and

$$
T^{k}:=\left(\begin{array}{ll}
1 & 0 \\
k & 1
\end{array}\right) \in \mathrm{GL}(2, \mathbb{Z}) .
$$

Let $\ell \subset \mathbb{R}^{2}$ be a vertical line in the plane, not necessarily through the origin, which splits it into two half-spaces, and let $n \in \mathbb{Z}$. Fix an origin in $\ell$. Let $t_{\ell}^{n}: \mathbb{R}^{2} \rightarrow$ $\mathbb{R}^{2}$ be the identity on the left half-space and $T^{n}$ on the right half-space. By definition $t_{\ell}^{n}$ is piecewise affine. Let $\ell_{i}$ be a vertical line through the focus-focus value $c_{i}=$ $\left(x_{i}, y_{i}\right)$, where $1 \leq i \leq m_{f}$, and for any tuple $\vec{n}:=\left(n_{1}, \ldots, n_{m_{f}}\right) \in \mathbb{Z}^{m_{f}}$ we set $t_{\vec{n}}:=t_{\ell_{1}}^{n_{1}} \circ \cdots \circ t_{\ell_{m_{f}}}^{n_{m_{f}}}$. The map $t_{\vec{n}}$ is piecewise affine.

A convex polygonal set $\Delta$ is the intersection in $\mathbb{R}^{2}$ of (finitely or infinitely many) closed half-planes such that on each compact subset of the intersection there is at most a finite number of corner points. We say that $\Delta$ is rational if each edge is directed along a vector with rational coefficients. For brevity, in this paper we usually write "polygon" (or "convex polygon") instead of "convex polygonal set". Note that the word polygon is commonly used to refer to the convex hull of a finite set of points in $\mathbb{R}^{2}$ which is a compact set (this is not necessarily the case in algebraic geometry, e.g., Newton polygons).

6.3.3. The polygon invariant. Let $B_{r}:=\operatorname{Int}(B) \backslash\left\{c_{1}, \ldots, c_{m_{f}}\right\}$, which is precisely the set of regular values of $F$. Given a sign $\epsilon_{i} \in\{-1,+1\}$, let $\ell_{i}^{\epsilon_{i}} \subset \ell_{i}$ be the vertical half-line starting at $c_{i}$ and extending in the direction of $\epsilon_{i}$ : upwards if $\epsilon_{i}=1$, downwards if $\epsilon_{i}=-1$. Let $\ell^{\vec{\epsilon}}:=\bigcup_{i=1}^{m_{f}} \ell_{i}^{\epsilon_{i}}$. In [108, Th. 3.8] it was shown that:

Theorem 6.3. For $\vec{\epsilon} \in\{-1,+1\}^{m_{f}}$ there exists a homeomorphism $f=f_{\epsilon}: B \rightarrow$ $\mathbb{R}^{2}$, modulo a left composition by a transformation in $\mathfrak{I}$, such that $\left.f\right|_{\left(B \backslash \ell^{\vec{\epsilon}}\right)}$ is a diffeomorphism into the image of $f, \Delta:=f(B)$, which is a rational convex polygon, $\left.f\right|_{\left(B_{r} \backslash \ell \vec{\epsilon}\right)}$ is affine (it sends the integral affine structure of $B_{r}$ to the standard structure of $\mathbb{R}^{2}$ ) and $f$ preserves $J$; i.e., $f(x, y)=\left(x, f^{(2)}(x, y)\right)$.

The map $f$ satisfies further properties 87 , which are relevant for the uniqueness proof. In order to arrive at $\Delta$, one cuts $F(M) \subset \mathbb{R}^{2}$ (see Figure 6.1 for a possible image $F(M)$ ) along each of the vertical half-lines $\ell_{i}^{\epsilon_{i}}$. Then the resulting image becomes simply connected, and thus there exists a global 2-torus action on the preimage of this set. The polygon $\Delta$ is just the closure of the image of a toric momentum map corresponding to this torus action.

We can see that this polygon is not unique. The choice of the "cut direction" is encoded in the signs $\epsilon_{j}$, and there remains some freedom for choosing the toric momentum map. Precisely, the choices and the corresponding homeomorphisms $f$ are the following:

(a) An initial set of action variables $f_{0}$ of the form $(J, K)$ near a regular Liouville torus in [108, Step 2, proof of Th. 3.8]. If we choose $f_{1}$ instead of $f_{0}$, we get a polygon $\Delta^{\prime}$ obtained by left composition with an element of $\mathfrak{I}$. Similarly, if we choose $f_{1}$ instead of $f_{0}$, we obtain $f$ composed on the left with an element of $\mathfrak{I}$.

(b) A tuple $\vec{\epsilon}$ of 1 and -1 . If we choose $\overrightarrow{\epsilon^{\prime}}$ instead of $\vec{\epsilon}$, we get $\Delta^{\prime}=t_{\vec{u}}(\Delta)$ with $u_{i}=\left(\epsilon_{i}-\epsilon_{i}^{\prime}\right) / 2$, by [108, Proposition 4.1, expression (11)]. Similarly instead of $f$, we obtain $f^{\prime}=t_{\vec{u}} \circ f$. 
Once $f_{0}$ and $\vec{\epsilon}$ have been fixed as in (a) and (b), respectively, then there exists a unique toric momentum map $\mu$ on

$$
M_{r}:=F^{-1}\left(\operatorname{Int}(B) \backslash \bigcup \ell_{j}^{\epsilon_{j}}\right)
$$

which preserves the foliation $\mathcal{F}$, and coincides with $f_{0} \circ F$ where they are both defined. Then, necessarily, the first component of $\mu$ is $J$, and we have $\overline{\mu\left(M_{r}\right)}=\Delta$.

We need now for our purposes to formalize choices (a) and (b) in a single geometric object. The details of how to do this have appeared in 88 . We will simply say that essentially this object consists of the convex polygon itself together with a collection of oriented cuts as in Figure 8.1 below. We call this object a weighted polygon, and denote it by $\Delta_{\mathrm{w}}$. The cuts are a collection of vertical lines that go through the singularities. The actual object is actually more complex, as it is defined as an equivalence class of such polygon considered as a part of a larger space of polygons on which two groups act nontrivially. These groups are $\{-1,1\}^{m_{f}}$ and $\mathfrak{I}$. The actual invariant is then denoted by $\left[\Delta_{\mathrm{w}}\right]$.

\section{MORE SYMPLECTIC INVARIANTS OF SEMITORIC SYSTEMS}

In [87, Theorem 6.2], starting from a given semitoric integrable system on a 4-manifold, the authors constructed a collection of five symplectic invariants associated with it and proved that these completely determine the integrable system up to global isomorphisms of semitoric systems. Let $M_{1}, M_{2}$ be symplectic 4-manifolds equipped with semitoric integrable systems $\left(J_{1}, H_{1}\right)$ and $\left(J_{2}, H_{2}\right)$. An isomorphism between these integrable systems is a symplectomorphism $\varphi: M_{1} \rightarrow M_{2}$ such that $\varphi^{*}\left(J_{2}, H_{2}\right)=\left(J_{1}, f\left(J_{1}, H_{1}\right)\right)$ for some smooth function $f$ such that $\frac{\partial f}{\partial H_{1}}$ nowhere vanishes.

We recall the definition of the invariants that we assigned to a semitoric integrable system in our previous paper [87, to which we refer for further details. Then we state the uniqueness theorem proved therein.

7.1. Taylor series invariant. We assume that the critical fiber $\mathcal{F}_{m}:=F^{-1}\left(c_{i}\right)$ contains only one critical point $m$, which according to Nguyên Tiên Zung [115] is a generic condition, and we let $\mathcal{F}$ denote the associated singular foliation. Moreover for simplicity, we will make an even stronger generic assumption: if $m$ is a focus-focus critical point for $F$, then $m$ is the unique critical point of the level set $J^{-1}(J(m))$. A semitoric system is simple if this generic assumption is satisfied.

These conditions imply that the values $J\left(m_{1}\right), \ldots, J\left(m_{m_{f}}\right)$ are pairwise distinct. We assume throughout the article that the critical values $c_{i}$ 's are ordered by their $J$-values: $J\left(m_{1}\right)<J\left(m_{2}\right)<\cdots<J\left(m_{m_{f}}\right)$. Let $\left(S_{i}\right)^{\infty}$ be a formal power series expansion (in two variables with vanishing constant term) corresponding to the integrable system given by $F$ at the critical focus-focus point $c_{i}$; see Theorem 5.4 We say that $\left(S_{i}\right)^{\infty}$ is the Taylor series invariant of $(M, \omega,(J, H))$ at the focus-focus point $c_{i}$. 
7.2. The volume invariant. Consider a focus-focus critical point $m_{i}$ whose image by $(J, H)$ is $c_{i}$, and let $\Delta$ be a rational convex polygon corresponding to the system $(M, \omega,(J, H))$. If $\mu$ is a toric momentum map for the system $(M, \omega,(J, H))$ corresponding to $\Delta$, then the image $\mu\left(m_{i}\right)$ is a point in the interior of $\Delta$, along the line $\ell_{i}$. We proved in [87] that the vertical distance

$$
h_{i}:=\mu\left(m_{i}\right)-\min _{s \in \ell_{i} \cap \Delta} \pi_{2}(s)>0
$$

is independent of the choice of momentum map $\mu$. Here $\pi_{2}: \mathbb{R}^{2} \rightarrow \mathbb{R}$ is $\pi_{2}(x, y)=y$. The reasoning behind writing the word "volume" in the name of this invariant is that it has the following geometric interpretation: the singular manifold $Y_{i}=$ $J^{-1}\left(c_{i}\right)$ splits into $Y_{i} \cap\left\{H>H\left(m_{i}\right)\right\}$ and $Y_{i} \cap\left\{H<H\left(m_{i}\right)\right\}$, and $h_{i}$ is the Liouville volume of $Y_{i} \cap\left\{H<H\left(m_{i}\right)\right\}$.

7.3. The twisting-index invariant. This is a subtle invariant of semitoric systems; it quantifies the dynamical complexity of the system at a global level, while involving the behavior near all of the focus-focus singularities of the system simultaneously.

The twisting index expresses the fact that there is, in a neighborhood of any focus-focus critical point $c_{i}$, a privileged toric momentum map $\nu$. This momentum map, in turn, is due to the existence of a unique hyperbolic radial vector field in a neighborhood of the focus-focus fiber. Therefore, one can view the twisting index as a dynamical invariant. Since any semitoric polygon defines a (generalized) toric momentum map $\mu$, we will be able to define the twisting index as the integer $k_{i} \in \mathbb{Z}$ such that

$$
\mathrm{d} \mu=T^{k_{i}} \mathrm{~d} \nu
$$

(Recall formula (6.1) for the formula of $T^{k_{i}}$.) We could have equivalently defined the twisting indices by comparing the privileged momentum maps at different focusfocus points.

The precise definition of $k_{i}$ requires some care, which we explain now. Let $\Delta_{\mathrm{w}}$ be the weighted polygon associated to $M$, which consists of the polygon $\Delta$ plus a collection of oriented vertical lines $\ell_{j}$, where the orientation of each line is given by \pm 1 signs $\epsilon_{j}, j=1, \ldots, m_{f}$.

Let $\ell:=\ell_{i}^{\epsilon_{i}} \subset \mathbb{R}^{2}$ be the vertical half-line starting at $c_{i}$ and pointing in the direction of $\epsilon_{i} e_{2}$, where $e_{1}, e_{2}$ are the canonical basis vectors of $\mathbb{R}^{2}$.

By Eliasson's theorem, there is a neighbourhood $W=W_{i}$ of the focus-focus critical point $m_{i}=F^{-1}\left(c_{i}\right)$, a local symplectomorphism $\phi:\left(\mathbb{R}^{4}, 0\right) \rightarrow W$, and a local diffeomorphism $g$ of $\left(\mathbb{R}^{2}, 0\right)$ such that $F \circ \phi=g \circ q$, where $q$ is given by (5.2).

Since $q_{2} \circ \phi^{-1}$ has a $2 \pi$-periodic Hamiltonian flow, it is equal to $J$ in $W$, up to a sign. Composing if necessary $\phi$ by $(x, \xi) \mapsto(-x,-\xi)$, one can assume that $q_{1}=J \circ \phi$ in $W$, i.e., $g$ is of the form $g\left(q_{1}, q_{2}\right)=\left(q_{1}, g_{2}\left(q_{1}, q_{2}\right)\right)$. Upon composing $\phi$ with $(x, y, \xi, \eta) \mapsto(-\xi,-\eta, x, y)$, which changes $\left(q_{1}, q_{2}\right)$ into $\left(-q_{1}, q_{2}\right)$, one can assume that $\frac{\partial g_{2}}{\partial q_{2}}(0)>0$. In particular, near the origin, $\ell$ is transformed by $g^{-1}$ into the positive imaginary axis if $\epsilon_{i}=1$ or the negative imaginary axis if $\epsilon_{i}=-1$.

Let us now fix the origin of angular polar coordinates in $\mathbb{R}^{2}$ on the positive imaginary axis. Let $V=F(W)$, and define $\tilde{F}=\left(H_{1}, H_{2}\right)=g^{-1} \circ F$ on $F^{-1}(V)$ (notice that $H_{1}=J$ ). 


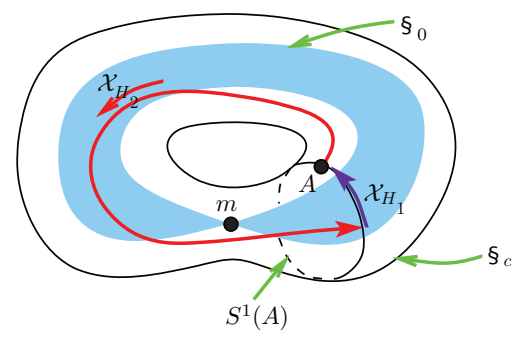

FIgURE 7.1. Singular foliation near the leaf $\Lambda_{0}=\mathcal{F}_{m}$, where $S^{1}(A)$ denotes the $S^{1}$-orbit generated by $H_{1}=J$.

Recall that near any regular torus there exists a Hamiltonian vector field $\mathcal{H}_{p}$, whose flow is $2 \pi$-periodic, defined by

$$
2 \pi \mathcal{H}_{p}=\left(\tau_{1} \circ \tilde{F}\right) \mathcal{H}_{H_{1}}+\left(\tau_{2} \circ \tilde{F}\right) \mathcal{H}_{J},
$$

where $\tau_{1}$ and $\tau_{2}$ are functions on $\mathbb{R}^{2} \backslash\{0\}$ satisfying (5.3), with $\sigma_{2}(0)>0$. In fact $\tau_{1}$ is multivalued, but we determine it completely in polar coordinates with angle in $[0,2 \pi)$ by requiring continuity in the angle variable and $\sigma_{1}(0) \in[0,2 \pi)$. In case $\epsilon_{i}=1$, this defines $\mathcal{H}_{p}$ as a smooth vector field on $F^{-1}(V \backslash \ell)$.

In case $\epsilon_{i}=-1$, we keep the same $\tau_{1}$-value on the negative imaginary axis, but extend it by continuity in the angular interval $[\pi, 3 \pi)$. In this way $\mathcal{H}_{p}$ is again a smooth vector field on $F^{-1}(V \backslash \ell)$.

Let $\mu$ be the generalized toric momentum map associated to $\Delta$. On $F^{-1}(V \backslash \ell)$, $\mu$ is smooth, and its components $\left(\mu_{1}, \mu_{2}\right)=\left(J, \mu_{2}\right)$ are smooth Hamiltonians whose vector fields $\left(\mathcal{H}_{J}, \mathcal{H}_{\mu_{2}}\right)$ are tangent to the foliation, have a $2 \pi$-periodic flow, and are a.e. independent. Since the couple $\left(\mathcal{H}_{J}, \mathcal{H}_{p}\right)$ shares the same properties, there must be a matrix $A \in \mathrm{GL}(2, \mathbb{Z})$ such that $\left(\mathcal{H}_{J}, \mathcal{H}_{\mu_{2}}\right)=A\left(\mathcal{H}_{J}, \mathcal{H}_{p}\right)$. This is equivalent to saying that there exists an integer $k_{i} \in \mathbb{Z}$ such that $\mathcal{H}_{\mu_{2}}=k_{i} \mathcal{H}_{J}+\mathcal{H}_{p}$.

It was shown in [87, Proposition 5.4] that $k_{i}$ is well defined, i.e., it does not depend on choices. The integer $k_{i}$ is called the twisting index of $\Delta_{\mathrm{w}}$ at the focusfocus critical value $c_{i}$.

It was shown in [87, Lemma 5.6] that there exists a unique smooth function $H_{p}$ on $F^{-1}(V \backslash \ell)$ with Hamiltonian vector field $\mathcal{H}_{p}$ and such that $\lim _{m \rightarrow m_{i}} H_{p}=0$. The toric momentum map $\nu:=\left(J, H_{p}\right)$ is called the privileged momentum map for $(J, H)$ around the focus-focus value $c_{i}$. If $k_{i}$ is the twisting index of $c_{i}$, one has $\mathrm{d} \mu=T^{k_{i}} \mathrm{~d} \nu$ on $F^{-1}(V)$. However, the twisting index does depend on the polygon $\Delta$. Thus, since we want to define an invariant of the initial semitoric system, we need to quotient-out by the natural action of groups $G_{m_{f}} \times \mathfrak{I}$; because this is a rather technical task, we refer to [87, p. 580] for details.

It was shown in [87, Proposition 5.8] that if two weighted polygons $\Delta_{\mathrm{w}}$ and $\Delta_{\text {weight }}^{\prime}$ lie in the same $G_{m_{f}}$-orbit, then the twisting indices $k_{i}, k_{i}^{\prime}$ associated to $\Delta_{\mathrm{w}}$ and $\Delta_{\text {weight }}^{\prime}$ at their respective focus-focus critical values $c_{i}, c_{i}^{\prime}$ are equal.

To a semitoric system we associate what we call the twisting-index invariant, which is nothing but the tuple $\left(\Delta_{\mathrm{w}}, \mathbf{k}\right)$ consisting of the polygon $\Delta$ labeled by the tuple twisting indices $\mathbf{k}=\left(k_{j}\right)_{j=1}^{m_{f}}$. Actually, as explained above, one needs to take into consideration the group actions of $G_{m_{f}}$ and $\mathfrak{I}$, so the twisting-index invariant associated to the semitoric system is an equivalence class $\left[\left(\Delta_{\mathrm{w}}, \mathbf{k}\right)\right]$ under a twisted 
action of $G_{m_{f}}$ and $\mathfrak{I}$. The formula for this action is long and we choose to not write it here, but details appear in 87 .

7.4. Example. In the case of the coupled spin-oscillator, the twisting-index invariant does not appear because there is only one focus-focus point. So in addition to the Taylor series invariant (of which as we said one can compute its linear approximation), the height invariant and the polygon invariant are easy to compute. They are explicitly given in Figure 8.2 in the next section.

\section{Global symplectic theory of Semitoric Systems}

8.1. First global result: uniqueness. The symplectic invariants constructed in 87. for a given 4-dimensional semitoric integrable system are the following:

(i) The number of singularities invariant. An integer $m_{f}$ counting the number of isolated singularities.

(ii) The singularity type invariant. A collection of $m_{f}$ infinite formal Taylor series on two variables which classifies locally the type of each (focus-focus) singularity.

(iii) The polygon invariant. The equivalence class of a weighted rational convex $\Delta_{\mathrm{w}}$ consisting of a convex polygon $\Delta$ and the collection of vertical lines $\ell_{j}$ crossing it, where $\ell_{j}$ is oriented upwards or downwards depending on the sign of $\epsilon_{j}, j=1, \ldots, m_{f}$.

(iv) The volume invariant. $m_{f}$ numbers measuring volumes of certain submanifolds at the singularities.

(v) The twisting-index invariant. $m_{f}$ integers measuring how twisted the system is around singularities.

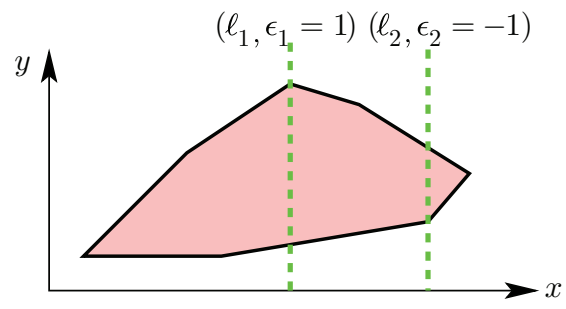

Figure 8.1. Weighted polygon $\left(\Delta,\left(\ell_{1}, \ell_{2}\right),(1,-1)\right)$.

This is a subtle invariant, which depends on the representative chosen in (iii). Here, we write $m_{f}$ to emphasize that the singularities that $m_{f}$ counts as focus-focus singularities. We then proved:

Theorem 8.1 (Pelayo and Vũ Ngọc [87]). Two semitoric systems $\left(M_{1}, \omega_{1},\left(J_{1}, H_{1}\right)\right)$ and $\left(M_{2}, \omega_{2},\left(J_{2}, H_{2}\right)\right)$ are isomorphic if and only if they have the same invariants (i)-(v), where an isomorphism is a symplectomorphism $\varphi: M_{1} \rightarrow M_{2}$ such that $\varphi^{*}\left(J_{2}, H_{2}\right)=\left(J_{1}, f\left(J_{1}, H_{1}\right)\right)$ for some smooth function $f$ such that $\frac{\partial f}{\partial H_{1}}$ nowhere vanishes. 


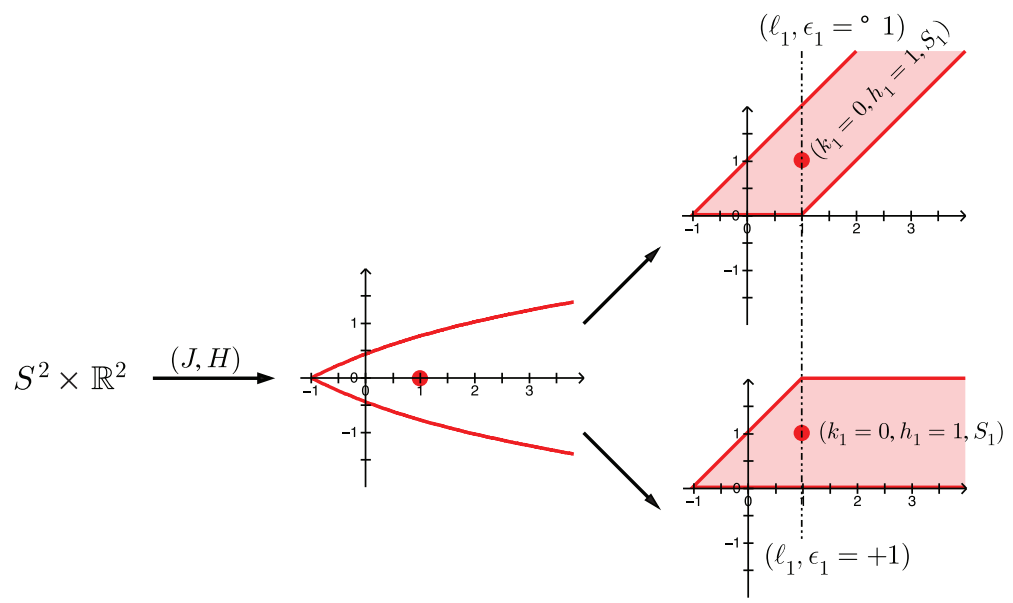

Figure 8.2. The coupled spin-oscillator example. The drawing at the center of the figure shows the image of the initial momentum map $F=(J, H)$. Its boundary is the parametrized curve $\left(j(s)=\frac{s^{2}-3}{2 s}, h(s)= \pm \frac{s^{2}-1}{2 s^{3 / 2}}\right), s \in[1, \infty)$. The image $F(M)$ of $F$ is the connected component of the origin. The system is a simple semitoric system with one focus-focus point whose image is $(1,0)$. The invariants are depicted on the right-hand side. Since $m_{f}=1$, the class of generalized polygons for this system consists of two polygons.

8.2. Second global result: existence. We have found that some restrictions on the symplectic invariants we have just defined must be imposed 88. Indeed, we call a "semitoric list of ingredients" the following collection of items:

(i) A nonnegative integer $m_{f}$.

(ii) An $m_{f}$-tuple of a formal Taylor series with vanishing constant term $\left(\left(S_{i}\right)^{\infty}\right)_{i=1}^{m_{f}} \in\left(\mathbb{R}[[X, Y]]_{0}\right)^{m_{f}}$.

(iii) A Delzant semitoric polygon $\left[\Delta_{\mathrm{w}}\right]$ of complexity $m_{f}$ consisting of a polygon $\Delta$ and vertical lines $\ell_{j}$ intersecting $\Delta$, each of which is oriented according to a sign $\epsilon_{j}= \pm 1$.

(iv) An $m_{f}$-tuple of numbers $\mathbf{h}=\left(h_{j}\right)_{j=1}^{m_{f}}$ such that $0<h_{j}<\operatorname{length}\left(\Delta \cap \ell_{i}\right)$.

(v) An equivalence class $\left[\left(\Delta_{\mathrm{w}}, \mathbf{k}\right)\right]$, where $\mathbf{k}=\left(k_{j}\right)_{j=1}^{m_{f}}$ is a collection of integers.

In the definition, the term $\mathbb{R}[[X, Y]]$ refers to the algebra of real formal power series in two variables, and $\mathbb{R}[[X, Y]]_{0}$ is the subspace of such series with vanishing constant term, and first term $\sigma_{1} X+\sigma_{2} Y$ with $\sigma_{2} \in[0,2 \pi)$. For the definition of Delzant semitoric polygon, which is somewhat involved, see [88, Section 4.2]. The main result of $[88$ is the following existence theorem:

Theorem 8.2 (Pelayo and Vũ Ngọc [88]). For each semitoric list of ingredients there exists a 4-dimensional simple semitoric integrable system with list of invariants equal to this list of ingredients.

The proof is involved, but the main idea of the proof is simple. We start with a representative of $\left[\Delta_{\mathrm{w}}\right]$ with all $\epsilon_{j}$ 's equal to +1 . The strategy is to construct the system locally and semiglobally around the singularities and around the regular 
parts to then perform symplectic gluing in order to obtain a semitoric system by constructing a suitable singular torus fibration above $\Delta \subset \mathbb{R}^{2}$.

Rather subtle analytical issues appear when gluing, and one eventually ends up with a system given by a momentum map which is not smooth along the cuts $\ell_{j}$. More concretely, first we construct a semitoric system over the part of the polygon away from the sets in the covering that contain the cuts $\ell_{j}^{+}$. Then we attach to this semitoric system the focus-focus fibrations, i.e., the models for the systems in a small neighborhood of the nodes (singularities). We use a symplectic gluing theorem to do this gluing (cf. 88, for a statement/proof).

Third, we continue to glue the local models in a small neighborhood of the cuts. The semitoric system is given by a proper toric map only in the preimage of the polygon away from the cuts. There is an analytically rather subtle issue near the cuts, and one has to change the momentum map carefully to make it smooth while preserving the structure of the system up to isomorphisms.

In the last step we prove that the system we have constructed has the right invariants. Here we have to appeal to the uniqueness theorem, as the equivalence class of the invariants may have shifted in the construction.

\section{Some open PRoblems}

9.1. Inverse spectral theory. Finding out how information from quantum completely integrable systems leads to information about classical systems is a fascinating "inverse" problem with very few precise results at this time.

The symplectic classification, in terms of concrete invariants described in Sections 6. 7, 8, serves as a tool to quantize semitoric systems. In Delzant's theory, the image of the momentum map, for a toric completely integrable action, completely

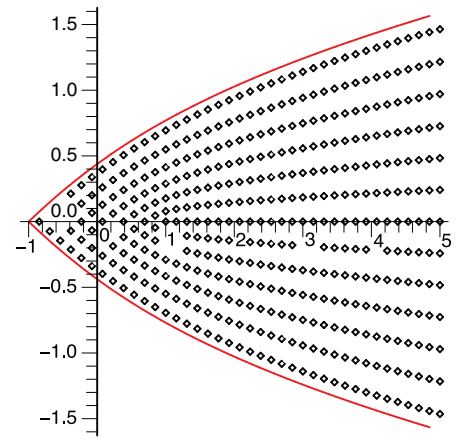

Figure 9.1. Sections 4 and 5 of Pelayo and Vũ Ngọc's article 89. are devoted to the spectral theory of the quantum coupled spinoscillator, and they are a first step towards proving this conjecture for spin-oscillators. The unbounded operators

$$
\hat{J}:=\operatorname{Id} \otimes\left(-\frac{\hbar^{2}}{2} \frac{\mathrm{d}^{2}}{\mathrm{~d} u^{2}}+\frac{u^{2}}{2}\right)+(\hat{z} \otimes \mathrm{Id}) \text { and } \hat{H}=\frac{1}{2}\left(\hat{x} \otimes u+\hat{y} \otimes\left(\frac{\hbar}{\mathrm{i}} \frac{\partial}{\partial u}\right)\right.
$$

on the Hilbert space $\mathcal{H} \otimes \mathrm{L}^{2}(\mathbb{R}) \subset \mathrm{L}^{2}\left(\mathbb{R}^{2}\right) \otimes \mathrm{L}^{2}(\mathbb{R})$ are self-adjoint and commute, and they define the quantum spin-oscillator. Their joint spectrum is depicted in this figure. 
determines the system. In the quantum theory, the image of the momentum map is replaced by the joint spectrum. Can one determine the underlying classical system from the joint spectrum of the associated quantum system? In this vast, essentially unexplored program, one can ask the less ambitious but still spectacular question: Given a quantum integrable system, depending on a semiclassical parameter $\hbar$ and whose semiclassical limit $(J, H)$ is semitoric, does the knowledge of the semiclassical joint spectra for all values of $\hbar$ determine the underlying classical system $(J, H)$ ?

Conjecture 9.1. A semitoric system $F=(J, H)$ is determined, up to symplectic equivalence, by its semiclassical joint spectrum (i.e., the collection of points $(\lambda, \nu) \in$ $\mathbb{R}^{2}$, where $\lambda$ is an eigenvalue of $\hat{J}$ and $\nu$ is an eigenvalue of $\hat{H}$ restricted to the $\lambda$ eigenspace of $\hat{J}$, as $\hbar \rightarrow 0$ ). Moreover, from any such semiclassical spectrum one can explicitly construct the associated semitoric system.

The strategy to prove this is clear: given the joint spectrum, detect in it the symplectic invariants. Once we have computed the symplectic invariants, we can symplectically recover the integrable system by [87, 88, and hence the quantum system. The authors have done this for the coupled spin-oscillator [89. The method to recover the symplectic invariants from the joint spectrum combines microlocal analysis and Lie theory. Recovering the polygon invariant is probably the easiest and most pictorial procedure, as long as one stays on a heuristic level. Making the heuristic rigorous should be possible along the lines of the toric case explained in [110] and [111.

The convex hull of the resulting set is a rational, convex polygonal set, depending on $\hbar$. Since the semiclassical affine structure is an $\hbar$-deformation of the classical affine structure, we see that, as $\hbar \rightarrow 0$, this polygonal set converges to the semitoric polygon invariant.

9.2. Mirror symmetry. When dealing with semitoric systems, we are in a situation where the moment map $(J, H)$ is a torus fibration with singularities, and

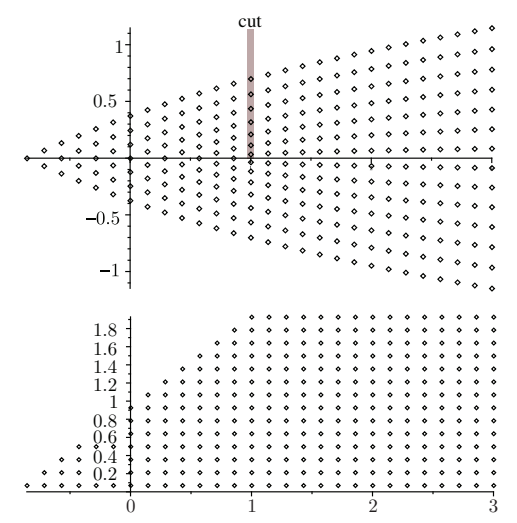

FiguRE 9.2. Recovering the polygon invariant. The top picture is the joint spectrum of $(\hat{J}, \hat{H})$. In the bottom picture, we have developed the joint eigenvalues into a regular lattice. One can easily check in this illustration that the number of eigenvalues in each vertical line in the same in both pictures. 
its base space becomes endowed with a singular integral affine structure. These same affine structures appear as a central ingredient in the work of Kontsevich and Soibelman 67]. These structures have been studied in the context of integrable systems (in particular by Nguyên Tiên Zung [115]), but they also became a central concept in the works by Symington [100] and Symington and Leung [71] in the context of symplectic geometry and topology, and by Gross and Siebert [52, 53, 54, 55], Castaño-Bernard [15, and Castaño-Bernard and Matessi [16, 17, among others, in the context of mirror symmetry and algebraic geometry. In fact the polygon invariant could have been expressed in terms of this affine structure. It will be interesting to interpret the results of this paper in the context of mirror symmetry; at the least the classification of semitoric systems would give a large class of interesting examples. We hope to explore these ideas in the future.

9.3. Higher dimensions. It is natural to want to extend our 4-dimensional classification results to higher dimensions. We believe this is a very difficult problem in general. Physically, there is no reason for dimension 4 to be more or less relevant than higher dimensions. The fact is that some of the results that our classification uses (primarily those of Vũ Ngọc, but not exclusively) are four-dimensional; but in principle there should be extensions to higher dimensions, as the proofs do not involve tools that are specific to dimension 4 .

9.4. Lagrange top equations. The heavy top equations in body representation are known to be Hamiltonian on $\mathfrak{s e}(3)^{*}$. These equations describe a classical Hamiltonian system with two degrees of freedom on the magnetic cotangent bundle

$\mathrm{T} S_{\left\|\Gamma_{0}\right\|}^{2}$. This system with two degrees of freedom has a conserved integral but it does not have, generically, additional integrals. However, in the Lagrange case, one can find one additional integral which makes the system completely integrable. It is classically known that the Lagrange heavy top is integrable. Moreover, one can check that it is semitoric, but it is given by a nonproper momentum map.

Problem 9.2. Develop the theory of semitoric systems $F=(J, H): M \rightarrow \mathbb{R}^{2}$ when the component $J$ generating a Hamiltonian circle action is not proper, but $F$ is proper.

The authors' general theory does not cover the case stated in Problem 9.2 but many of techniques do extend at least to the case when $F$ is a proper map. We have been exploring this case in 85.

\section{ABOUT THE AUTHORS}

The first author is a member of IAS and an assistant professor at Washington University. Recently, he was recipient of the Prize Rubio de Francia and an NSF CAREER Award. The second author is a professor of mathematics at the Université de Rennes. In 2010 he was elected a Member of the Institut Universitaire de France.

\section{ACKNOWLEDGMENTS}

We thank the anonymous referees for many helpful comments and remarks. The first author was partly supported by NSF Postdoctoral Fellowship DMS-0703601, NSF Grant DMS-0965738, a Leibniz Fellowship from the Oberwolfach Foundation, and MSRI and IAS memberships. He gratefully acknowledges the Mathematical Sciences Research Institute in Berkeley for their hospitality during the academic 
year 2009-2010, and the Mathematisches Forschungsinstitut Oberwolfach for their hospitality during part of the summer of 2010. The second author was partly supported by an ANR "Programme Blanc". Finally, thanks are due to Michael VanValkenburgh for his careful reading of a preliminary version.

\section{REFERENCES}

[1] R. Abraham and J. E. Marsden: Foundation of Mechanics, Second edition, revised and enlarged. With the assistance of Tudor Ratiu and Richard Cushman. Benjamin/Cummings Publishing Co., Inc., Advanced Book Program, Reading, Mass., 1978. MR515141 (81e:58025)

[2] K. Ahara and A. Hattori: 4-dimensional symplectic $S^{1}$-manifolds admitting a moment map, J. Fac. Sci. Univ. Tokyo Sect. IA, Math 38 (1991), 251-298. MR1127083 (93b:58048)

[3] A. Alekseev: On Poisson actions of compact Lie groups on symplectic manifolds, J. Diff. Geometry, 45 (1997), pp. 241-256. MR1449971 (99b:58086)

[4] V. I. Arnold: A theorem of Liouville concerning integrable problems of dynamics. Siberian Math. J 4, 1963. MR0147742(26:5256)

[5] E. Assémat, K. Efstathiou, M. Joyeux, and D. Sugny: Fractional bidromy in the vibrational spectrum of hocl. Phys. Rev. Letters, 104 (113002): (2010) 1-4.

[6] M. Atiyah: Convexity and commuting Hamiltonians. Bull. London Math. Soc. 14 (1982) 1-15. MR.642416 (83e:53037)

[7] M. Audin: Hamiltoniens périodiques sur les variétés symplectiques compactes de dimension 4, Géometrie symplectique et mécanique, Proceedings 1988, C. Albert ed., Springer Lecture Notes in Math. 1416 (1990). MR.1047474 (91f:57013)

[8] M. Audin: The Topology of Torus Actions on Symplectic Manifolds, Birkhäuser, 1991. MR 1106194 (92m:57046)

[9] O. Babelon, L. Cantini, and B. Douçot: A semi-classical study of the Jaynes-Cummings model. (English summary) J. Stat. Mech. Theory Exp. 2009, no. 7, P07011.

[10] Y. Benoist: Actions symplectiques de groupes compacts. Geometriae Dedicata 89 (2002) 181-245, and Correction to "Actions symplectiques de groupes compacts", http://www.dma.ens.fr/ benoist. MR1890958 (2003e:53117)

[11] A. Bolsinov and A. Oshemkov: Singularities of integrable Hamiltonian systems. Topological Methods in the Theory of Integrable Systems, Cambridge Scientific Publ., 2006, 1-67. MR 2454549(2009m:37160)

[12] A. V. Bolsinov and A. T. Fomenko: Integrable Hamiltonian Systems; Geometry, Topology, Classification. Chapman \& Hall, 2004. Translated from the 1999 Russian original. MR2036760 (2004j:37106)

[13] A. V. Bolsinov, P. H. Richter and A. T. Fomenko: Loop molecule method and the topology of the Kovalevskaya top, Sbornik: Mathematics 191 (2000) No. 2, 151-188. MR 1751773 (2001e:37072)

[14] B. Branham and H. Hofer: First steps towards a symplectic dynamics. arXiv:1102.3723

[15] R. Castaño-Bernard: Symplectic invariants of some families of Lagrangian $T^{3}$-fibrations, $J$. Symplectic Geometry 2, (2004), no. 3, 279-308. MR2131638 (2005k:53167)

[16] R. Castaño-Bernard and D. Matessi: Some piece-wise smooth Lagrangian fibrations, Rend. Semin. Mat. Univ. Politec. Torino 63 (2005), no.3, 223-253. MR2201567 (2006j:53115)

[17] R. Castaño-Bernard and D. Matessi: Lagrangian 3-torus fibrations, J. Diff. Geometry 81 (2009) 483-573. MR.2487600(2010e:53146)

[18] A.-M. Charbonnel: Comportement semi-classique du spectre conjoint d'opérateurs pseudodifférentiels qui commutent. Asymptotic Analysis 1 (1988) 227-261. MR962310 (89j:35100)

[19] M. S. Child, T. Weston, and J. Tennyson: Quantum monodromy in the spectrum of H2O and other systems: new insight into the level structure of quasi-linear molecules. Mol. Phys., 96 (3) (1999) 371-379.

[20] L. Charles: Quasimodes and Bohr-Sommerfeld conditions for the Toeplitz operators. Comm. Partial Differential Equations, 28(9-10), 2003. MR2001172(2005d:53142)

[21] Y. Colin de Verdière: Spectre conjoint d'opérateurs pseudo-différentiels qui commutent II. Math. Z., 171 (1980) 51-73. MR566483 (81i:58046)

[22] Y. Colin de Verdière and B. Parisse: Équilibre instable en régime semi-classique I : Concentration microlocale. Comm. Partial Differential Equations, 19 (9-10) (1994) 1535-1563. 
[23] Y. Colin de Verdière and B. Parisse: Équilibre instable en régime semi-classique II : Conditions de Bohr-Sommerfeld. Ann. Inst. H. Poincaré. Phys. Théor., 61(3) (1994) 347-367. MR:1311072 (97a:81041)

[24] Y. Colin de Verdière and J. Vey: Le lemme de Morse isochore. Topology, 18 (1979) 283-293. $\operatorname{MR} 551010$ (80k:57059)

[25] Y. Colin de Verdière: Singular Lagrangian manifolds and semiclassical analysis. Duke Math. J., 116 (2003) 263-298. MR1953293 (2003j:53122)

[26] Y. Colin de Verdière and S. Vũ Ngọc: Singular Bohr-Sommerfeld rules for 2D integrable systems. Ann. Sci. École Norm. Sup. 36 (2003) 1-55. MR.1987976 (2004h:58040)

[27] R. H. Cushman and L. M. Bates: Global Aspects of Classical Integrable Systems. Birkhaüser Verlag, Basel, 1997. MR 1438060 (98a:58083)

[28] G. Darboux: Sur le problème de Pfaff, Bulletin des Sciences mathéma. et astrono., 2 série, t. VI; I88z, (1882) 1-46.

[29] R. de la Llave: A tutorial on KAM theory. Smooth ergodic theory and its applications (Seattle, WA, 1999), 175-292, Proc. Sympos. Pure Math., 69, Amer. Math. Soc., Providence, RI, 2001. MR1858536 (2002h:37123)

[30] T. Delzant: Hamiltoniens périodiques et image convexe de l'application moment. Bull. Soc. Math. France 116 (1988) 315-339. MR984900 (90b:58069)

[31] J. J. Duistermaat: On global action-angle variables. Comm. Pure Appl. Math., 33, (1980) 687-706. MR596430 (82d:58029)

[32] J. J. Duistermaat and Á. Pelayo: Symplectic torus actions with coisotropic principal orbits, Annales de l'Institut Fourier 57 (2007) 2239-2327. MR2394542 (2009g:53048)

[33] J. J. Duistermaat and Á. Pelayo: Reduced phase space and toric variety coordinatizations of Delzant spaces, Math. Proc. Cambr. Phil. Soc. 146 (2009) 695-718. MR2496353 (2010g:53161)

[34] J. J. Duistermaat and Á. Pelayo: Complex structures on four-manifolds with symplectic two-torus actions. Intern. J. of Math. 22 (2011) 449-463.

[35] J. J. Duistermaat and Á. Pelayo: Topology of symplectic torus actions with symplectic orbits. Revista Matemática Complutense 24 (2011) 59-81.

[36] J. J. Duistermaat: Personal communication, December 2009.

[37] J. P. Dufour and P. Molino: Compactification d'actions de $\mathbb{R}^{n}$ et variables actions-angles avec singularités. In Dazord and Weinstein, editors, Séminaire Sud-Rhodanien de Géométrie à Berkeley, volume 20, pp. 151-167. MSRI, 1989. MR1104924 (92b:58066)

[38] J.-P. Dufour, P. Molino, and A. Toulet: Classification des systèmes intégrables en dimension 2 et invariants des modèles de Fomenko. C. R. Acad. Sci. Paris Sér. I Math., 318 (1994) 949-952. MR1278158 (95d:58053)

[39] H. Dullin and S. Vũ Ngọc: Vanishing twist near focus-focus points. Nonlinearity 17 (2004), no. 5, 1777-1785. MR2086150 (2005e:37121)

[40] H. Dullin and S. Vũ Ngọc: Symplectic invariants near hyperbolic-hyperbolic points. Regul. Chaotic Dyn. 12 (2007), no. 6, 689-716. MR2373167(2009a:37105)

[41] Y. Eliashberg and L. Polterovich: Symplectic quasi-states on the quadric surface and Lagrangian submanifolds, arXiv:1006.2501.

[42] L. H. Eliasson. Normal forms for hamiltonian systems with Poisson commuting integrals elliptic case. Comment. Math. Helv. 65 (1990) 4-35. MR1036125(91d:58223)

[43] L. H. Eliasson: Hamiltonian systems with Poisson commuting integrals, PhD thesis, University of Stockholm, 1984.

[44] N. J. Fitch, C. A. Weidner, L. P. Parazzoli, H. R. Dullin, and H. J. Lewandowski: Experimental demonstration of classical hamiltonian monodromy in the $1: 1: 2$ resonant elastic pendulum. Phys. Rev. Lett., (2009) (034301).

[45] H. Flaschka and T. S. Ratiu: A convexity theorem for Poisson actions of compact Lie groups, Ann. Sci. Ecole Norm. Sup., 29 (6) (1995), pp. 787-809. MR1422991 (98a:58068)

[46] A. T. Fomenko: Topological Classification of Integrable Systems, volume 6 of Advances in Soviet Mathematics. AMS, 1991. MR1141218 (92i:58003)

[47] T. Frankel: Fixed points and torsion on Kähler manifolds, Ann. Math. 70 (1959), 1-8. MR 0131883 (24:A1730)

[48] K. E. Feldman: Hirzebruch genera of manifolds supporting a Hamiltonian circle action. (Russian) Uspekhi Mat. Nauk 56(5) (2001), 187-188; translation in Russian Math. Surveys 56(5) (2001), 978-979. MR1892568(2003a:57062) 
[49] M. Garay: A rigidity theorem for Lagrangian deformations. Compos. Math. 141 (2005), no. 6, 1602-1614. MR2188452 (2006k:32057)

[50] A. Giacobbe: Convexity of multi-valued momentum maps, Geometriae Dedicata 111 (2005), 1-22. MR2155173(2006m:53133)

[51] V. L. Ginzburg: Some remarks on symplectic actions of compact groups, Math. Zeitschrift 210 (1992), 625-640. MR:1175727(93h:57053)

[52] M. Gross and B. Siebert: Affine manifolds, log structures, and mirror symmetry, Turkish J. Math. 27 (2003), 33-60. math.AG/0211094. MR1975331 (2004g:14041)

[53] M. Gross and B. Siebert: Mirror symmetry via logarithmic degeneration data I, J. Diff. Geometry 72 (2006), 169-338. MR2213573 (2007b:14087)

[54] M. Gross and B. Siebert: Mirror symmetry via logarithmic degeneration data II, J. of Alg. Geom. 19 (2010) 679-780. MR2669728

[55] M. Gross and B. Siebert: From real affine geometry to complex geometry, arXiv:math/ 0703822 .

[56] V. Guillemin and S. Sternberg: Convexity properties of the moment mapping. Invent. Math. 67 (1982) 491-513. MR664117 (83m:58037)

[57] V. V. Kalashnikov: Typical integrable Hamiltonian systems on a four-dimensional symplectic manifold. Izvest. Akad. Nauk SSSR, Ser. Matem. 62 (1998), 49-74. MR 1623822 (99g:58052)

[58] Y. Karshon: Periodic Hamiltonian flows on four-dimensional manifolds, Contact and Symplectic Geometry (ed. C.B. Thomas), INI Publications, No. 8, Cambridge Univ. Press (1996), pp. 43-47. MR1432457(98a:58069)

[59] Y. Karshon: Periodic Hamiltonian flows on four-dimensional manifolds. Memoirs Amer. Math. Soc. No. 672141 (1999), viii+71 pp. MR1612833 (2000c:53113)

[60] M. P. Kharlamov: Topologicheskii analiz integriruemykh zadach dinamiki tverdogo tela. (Russian) [Topological analysis of integrable problems of rigid body dynamics] Leningrad. Univ., Leningrad, 1988. 200 pp. MR948454(89i:58036)

[61] F. Kirwan: Convexity properties of the moment mapping. III. Invent. Math. 77 (1984) 547-552. MR759257 (86b:58042b)

[62] B. Kostant: Orbits, symplectic structures and representation theory. 1966 Proc. U.S.Japan Seminar in Differential Geometry (Kyoto, 1965) p. 71 Nippon Hyoronsha, Tokyo MR0213476 (35:4340)

[63] B. Kostant: Quantization and unitary representations. I. Prequantization. Lectures in modern analysis and applications, III, pp. 87-208. Lecture Notes in Math., Vol. 170, Springer, Berlin, 1970. MR0294568 (45:3638)

[64] B. Kostant and A. Pelayo: Geometric Quantization, Springer, to appear.

[65] J. Kedra, Y. Rudnyak and A. Tralle: Symplectically aspherical manifolds, J. Fixed Point Theory Appl. 3 (2008), 1-21. MR2402905 (2009b:57054)

[66] K. Kodaira: On the structure of compact analytic surfaces, I. Amer. J. Math. 86 (1964) 751-798. MR0187255(32:4708)

[67] M. Kontsevich and Y. Soibelman: Affine structures and non-Archimedean analytic spaces. The unity of mathematics, 321-385, Progr. Math., 244, Birkhäuser Boston, Boston, MA, 2006. MR 2181810 (2006j:14054)

[68] C. Laurent-Gengoux, E. Miranda and P. Vanhaecke: Action-angle coordinates for integrable systems on Poisson manifolds. Preprint, arxiv.0805.1679.

[69] L. M. Lerman and Ya. L. Umanskii: Structure of the Poisson action of $\mathbb{R}^{2}$ on a fourdimensional symplectic manifold. I, II. Selecta Math. Sov. 6 (1987) 365-396; 7 (1988) 3-48. MR 925264 (90g:58037)

[70] L. M. Lerman and Ya. L. Umanskii: Classification of four-dimensional integrable systems and the Poisson action of $\mathbb{R}^{2}$ in extended neighborhoods of simple singular points. I, II, III. Matem. Sbornik. 183 (12), (1992) 141-176; 184 (4), (1993) 103-138, 1993; 186 (1995) (10), 89-102. MR 1361596 (96m:58087)

[71] N. C. Leung and M. Symington: Almost toric symplectic four-manifolds. J. Symplectic Geom. 8 (2010) 143-187. MR2670163 (2011e:53146)

[72] Y. Lin and A. Pelayo: Non-Kähler symplectic manifolds with toric symmetries. Quart. J. Math. 62 (2011) 103-114.

[73] J. Liouville: Note sur l'intégration des équations différentielles de la Dynamique. J. Math. Pures Appl., 20 137-138 (1855). Présentée en 1853. 
[74] G. Lupton and J. Oprea: Cohomologically symplectic spaces: toral actions and the Gottlieb group, Trans. Amer. Math. Soc. 347(1) (1995), 261-288. MR1282893 (95f:57056)

[75] H. Mineur: Sur les systèmes mécaniques dans lesquels figurent des paramètres fonctions du temps. etude des systemes admettant $\mathrm{n}$ intégrales premies uniformes en involution. Extension à ces systèmes des conditions de quantification de Bohr-Sommerfeld. J. Ecole Polytechn., III (1937) (Cahier 1, Fasc. 2 et 3):173-191, 237-270.

[76] J. E. Marsden and T. S. Ratiu: Introduction to Mechanics and Symmetry, Texts in Applied Mathematics 17, second edition, second printing, Springer Verlag, New York, 2003. MR.1304682 (95i:58073)

[77] E. Miranda and Nguyên Tiên Zung: Equivariant normal for for non-degenerate singular orbits of integrable Hamiltonian systems. Ann. Sci. École Norm. Sup. (4), 37(6):819-839, 2004. MR 2119240 (2006c:37062)

[78] E. Miranda and S. Vũ Ngọc: A singular Poincaré lemma. Intern. Math. Res. Not. IMRN $2005127-45$.

[79] D. McDuff: The moment map for circle actions on symplectic manifolds, J. Geom. Phys. 5(2) (1988) 149-160. MR 1029424 (91c:58042)

[80] N. Nekhoroshev, D. Sadovskii and B. Zhilinskii: Fractional Hamiltonian monodromy, Ann. Henri Poincaré 7 (6) (2006) 1099-1211. MR2267061(2008f:37119)

[81] J.-P. Ortega and T. S. Ratiu: A symplectic slice theorem. Lett. Math. Phys. 59 (2002) 81-93. MR 1894237 (2003f:53152)

[82] J.-P. Ortega and T. S. Ratiu, Momentum Maps and Hamiltonian Reduction, Progress in Mathematics 222, Birkhäuser Boston, 2004. MR2021152 (2005a:53144)

[83] Á. Pelayo: Symplectic actions of 2-tori on 4-manifolds, Mem. Amer. Math. Soc. 204 (2010) no. 959 MR2640344

[84] Á. Pelayo and T. S. Ratiu: Applying Hodge theory to dectect Hamiltonian flows. Preprint. arXiv.1005.2163.

[85] Á. Pelayo, T. S. Ratiu and S. Vũ Ngọc: Singular Lagrangian fibrations of Hamiltonian systems. Preprint.

[86] Á. Pelayo and S. Tolman: Fixed points of symplectic periodic flows. Erg. Theory and Dyn. Syst. 31 (2011), in press.

[87] Á. Pelayo and S. Vũ Ngọc: Semitoric integrable systems on symplectic 4-manifolds. Invent. Math. 177 (2009) 571-597. MR2534101 (2011a:37110)

[88] Á. Pelayo and S. Vũ Ngọ: Constructing integrable systems of semitoric type. Acta Math. 206 (2011) 93-125.

[89] Á. Pelayo and S. Vũ Ngọc: Symplectic and spectral theory for spin-oscillators. Preprint. arXiv.1005.439.

[90] N. Roy: The geometry of nondegeneracy conditions in completely integrable systems. Corrected reprint of Ann. Fac. Sci. Toulouse Math. (6) 14 (2005), no. 4, 705-719 [ MR2188589]. Ann. Fac. Sci. Toulouse Math. (6) 15 (2006), no. 2, 383-397. MR2188589 (2006g:37086)

[91] H. Rüssmann. Über das Verhalten analytischer Hamiltonscher Differentialgleichungen in der Nähe einer Gleichgewichtlösung. Math. Ann., 154:285-300, 1964. MR0179409(31:3657)

[92] D. A. Sadovskií and B. Zhilinskií: Counting levels within vibrational polyads. J. Chem. Phys., 103 (24) (1995).

[93] D. A. Sadovskií and B. I. Zhilinskií. Monodromy, diabolic points, and angular momentum coupling. Phys. Lett. A, 256 (4) (1999) 235-244. MR1689376 (2000b:81027)

[94] C. Sevenheck and D. van Straten: Rigid and complete intersection Lagrangian singularities. Manuscripta Math. 114 (2004), no. 2, 197-209. MR2067793 (2005h:32075)

[95] J. P. Souriau: Quantification géométrique. Comm. Math. Phys. 1 (1966) 374-398. MR0207332 (34:7148)

[96] J. P. Souriau: Structure des Systèmes Dynamiques. Dunoud, Paris 1970. English translation by R.H. Cushman and G.M. Tuynman. Progress in Mathematics, 149. Birkhäuser Boston, 1997. MR 1461545 (98i:58103)

[97] P. Stefan. Accessible sets, orbits, and foliations with singularities. Proc. Lond. Math. Soc. 29 (1974) 699-713. MR0362395 (50:14837)

[98] I. Stewart: Quantizing the classical cat. Nature, 430 (2004), 731-732.

[99] M. Symington. Generalized symplectic rational blowdowns. Algebraic and Geometric Topology, 1(26):503-518, 2001. MR1852770 (2002g:57056) 
[100] M. Symington: Four dimensions from two in symplectic topology. pp. 153-208 in Topology and geometry of manifolds (Athens, GA, 2001). Proc. Sympos. Pure Math., 71, Amer. Math. Soc., Providence, RI, 2003. MR2024634(2005b:53142)

[101] J. R. Taylor: Classical Mechanics, University Science Books (2005).

[102] S. Tolman and J. Weitsman: On semifree symplectic circle actions with isolated fixed points, Topology 39 (2000), 299-309. MR 1722020 (2000k:53074)

[103] J. Toth: On the quantum expected values of integrable metric forms. J. Diff. Geometry, 52 327-374, 1999. MR 1758299 (2001j:58048)

[104] J. Toth and S. Zelditch: Riemannian manifolds with uniformly bounded eigenfunctions. Duke Math. J., 111(1):97-132, 2002. MR.1876442(2003d:58048)

[105] J. Vey: Sur certains systèmes dynamiques séparables. Amer. J. Math., 100:591-614, 1978. MR0501141 (58:18571)

[106] S. Vũ Ngọc: Bohr-Sommerfeld conditions for integrable systems with critical manifolds of focus-focus type. Comm. Pure Appl. Math., 53(2):143-217, 2000. MR 1721373 $(2001 \mathrm{~m}: 81081)$

[107] S. Vũ Ngọc: On semi-global invariants for focus-focus singularities. Top. 42 (2003), no. 2, 365-380. MR1941440 (2004b:37119)

[108] S. Vũ Ngọc: Moment polytopes for symplectic manifolds with monodromy. Adv. Math. 208 (2007), no. 2, 909-934. MR2304341(2008e:53169)

[109] S. Vũ Ngoc: Symplectic techniques for semiclassical integrable systems, Topological Methods in the Theory of Integrable Systems, Cambridge Scientific, 2006. MR 2454557|(2009m:37168)

[110] S. Vũ Ngọ: Systèmes Integrables Semi-Classiques : du Local au Global. Pan. et Synthèses SMF, 22, 2006.

[111] S. Vũ Ngọc: Symplectic inverse spectral theory for pseudodifferential operators. To appear in Geometric Aspects of Analysis and Mechanics, in honor of the 65th birthday of Hans Duistermaat; to be published by Birkhäuser, Boston, 2011.

[112] S. Vũ Ngọc and C. Wacheux: Smooth normal forms for integrable hamiltonian systems near a focus-focus singularity. hal preprint oai:hal.archives-ouvertes.fr: hal-00577205, arXiv: 1103.3282 .

[113] J. Williamson: On the algebraic problem concerning the normal form of linear dynamical systems. Amer. J. Math., 58 (1996) 141-163. MR.1507138

[114] Nguyên Tiên Zung: Kolmogorov condition for integrable systems with focus-focus singularities. Phys. Lett. A, 215 (1-2) (1996) 40-44. MR1396244(97e:58092)

[115] Nguyên Tiên Zung: Symplectic topology of integrable hamiltonian systems, I: ArnoldLiouville with singularities. Compositio Math., 101 (1996) 179-215. MR1389366 (97c:58052)

[116] Nguyên Tiên Zung: A note on degenerate corank-1 singularities of integrable Hamiltonian systems, Comment. Helv. Math. 75 (2000), 271-283. MR1774706 (2002e:37093)

[117] Nguyên Tiên Zung: Kolmogorov condition near hyperbolic singularities of integrable Hamiltonian systems. Regul. Chaotic Dyn. 12 (2007), no. 6, 680-688. MR2373157 (2009a:37110)

[118] Nguyên Tiên Zung: Convergence versus integrability in Birkhoff normal form: Ann. of Math. (2) 161 (2005), no. 1, 141-156. MR2150385 (2006i:37128)

[119] A. Weinstein: Poisson geometry of discrete series orbits, and momentum convexity for noncompact group actions, Letters in Mathematical Physics, 56 (1) (2001), 17-30. MR 1848163 (2002m:53130)

Department of Mathematics, Washington University, One Brookings Drive, Campus Box 1146, St. Louis, Missouri 63130-4899; And School of Mathematics, Institute for Advanced Study, Einstein Drive, Princeton, New Jersey 08540

E-mail address: apelayo@math.wustl.edu; apelayo@math.ias.edu

$U R L:$ http://www.math. wustl.edu/ apelayo/

Institut de Recherches Mathématiques de Rennes, Université de Rennes 1, Campus de Beaulieu, 35042 Rennes cedex, France

E-mail address: san.vu-ngoc@univ-rennes1.fr

$U R L:$ http://blogperso.univ-rennes1.fr/san.vu-ngoc/ 